\title{
Cybersicherheitsrecht im Gesundheitswesen
}

\section{Carsten Dochow*}

\begin{abstract}
Die aktuelle Cyber-Bedrohungslage für Einrichtungen des Gesundheitswesens, die infolge der forcierten Digitalisierung begünstigt wird, gibt Anlass für eine verstärkte Betrachtung des Cybersicherheitsrechts im Gesundheitswesen. Cybersicherheit bezieht sich dabei nicht nur auf den Schutz von personenbezogenen Daten, sondern nimmt ebenso die Funktionsfähigkeit von IT-Systemen der Einrichtungen sowie die Sicherheit und Vertrauenswürdigkeit bei allen Informationsverarbeitungsprozessen in den Blick. Sie gewinnt aufgrund der großen Abhängigkeit von verlässlichen Informations- und Kommunikationsstrukturen auch im Gesundheitsbereich zunehmend an Bedeutung. Der Beitrag gibt einen Überblick über die fragmentarisch geregelten Rechtsgrundlagen und befasst sich dabei auch mit der jüngeren Rechtssetzung. Seit dem 1.1.2022 gelten infolgedessen „neue" Verpflichtungen für Arztpraxen und Krankenhäuer.
\end{abstract}

\section{Verschärfte Cyber-Bedrohungslage im Gesundheitssektor}

Das Bundeskabinett hat am 8.9.2021 eine „Cybersicherheitsstrategie für Deutschland 2021“ beschlossen ${ }^{1}$. Diese bildet den strategischen Rahmen für das Handeln der Bundesregierung $^{2}$ für fünf Jahre und definiert übergreifende Leitlinien. Es werden vier Handlungsfelder mit zahlreichen strategischen Zielen bestimmt. Das Gesundheitswesen wird im Rahmen dieser allgemeinen Zielsetzungen als wertvolles „Asset“ eingeordnet ${ }^{3}$. Es sollen hier u.a. „Voraussetzungen für sichere elektronische Kommunikation" geschaffen, der „Schutz kritischer Infrastrukturen“" weiter verbessert und der Wirtschaftszweig Gesundheitswesen durch Erhöhung der IT-Sicherheit von Produkten (z.B. Digitale Gesundheitsanwendungen) gezielt gestärkt werden ${ }^{4}$.

Diese Zielsetzungen sind in Ansehung des rapide wachsenden Bedrohungspotenzials durch zunehmend zielgerichtete, technologisch ausgereiftere und komplexere Angriffe ${ }^{5}$ verständlich. Die „Bedrohungslage [...] ist gekennzeichnet durch eine deutliche sowohl qualitative als auch quantitative Zunahme von Cyberangriffen, eine wachsende Angriffsfläche und neuartige Bedrohungsszenarien. Zudem steigt die potenzielle Schadenshöhe" ${ }^{\text {" }}$. Das BSI hat zuletzt eine ,angespannte bis kritische“ IT-Sicherheitslage festgestellt $^{7}$. Insgesamt seien die Anzahl von Sicherheitsvorfällen und die rasante Entwicklung neuer und angepasster Angriffsmethoden besorgniserregend ${ }^{8}$. Gekennzeichnet sei die Lage durch ein eklatant erhöhtes Auftreten der Androhung von DDoS-Angriffen, Lösegelderpressung durch Ransomware sowie Schweigegelderpressungen?.

Besonders gefährdet sind durch diese Art der CyberKriminalität auch Arztpraxen ${ }^{10}$ und Krankenhäuser ${ }^{11}$. Die Bedrohungslage im Gesundheitswesen ist schon in den letzten 5 bis 6 Jahren sichtbarer geworden, seitdem im Jahr 2016 Störungen in Krankenhäusern durch „Locky“ bekannt wurden, infolgedessen Infrastrukturen vieler Kliniken stillgelegt waren. Im Jahr 2017 erfolgten WannaCryAngriffe auf Krankenhäuser-IT und im Jahr 2019 wurden Infektionen der IT-Infrastruktur von DRK-Kliniken mit der Ransomware „Sodinokibi“ und ein Befall des ITSystems des Klinikums Fürth mit der Malware „Emotet“ festgestellt ${ }^{12}$. Im Zusammenhang mit einem Cyberangriff

Dr. iur. Carsten Dochow,

Leiter Personal, Organisation und Datenschutz

der Bundesärztekammer,

Herbert-Lewin-Platz 1, 10623 Berlin, Deutschland auf das Universitätsklinikum Düsseldorf im Jahr 2020 steht wohl sogar der Tod einer Patientin ${ }^{13}$. Zuletzt waren in der COVID-19-Pandemie neben Kliniken ${ }^{14}$ auch verstärkt Hersteller von Impfstoffen oder COVID-19-Antigentests betroffen ${ }^{15}$. Im November 2020 lag die Zahl der erfolgreichen Attacken auf kritische Infrastrukturen im Gesundheitsbereich bei 43; im Vorjahr waren es noch $16^{16}$.

Die medizinischen Einrichtungen sind allerdings nicht nur direkt betroffen. Auch ihre Dienstleister sind Angriffen ausgesetzt. So war im November 2021 ein IT-Dienstleister, der viele Arztpraxen mit Software ausstattet, von einer Ransomware-Attacke betroffen; ebenso wie im Dezember 2021 der marktführende Hersteller von Anwendungssoftware für Arztpraxen, Apotheken, medizinischen Laboratorien sowie Krankenhäusern ${ }^{17}$. Zwar seien Infrastrukturen oder IT-Systeme der Praxen nicht direkt tangiert gewesen. Den Arztpraxen wurde indes empfohlen, unverzüg-

^) Es wird hier ausschließlich die persönliche Auffassung des Autors wiedergegeben.

1) BMI, Cybersicherheitsstrategie für Deutschland 2021, S. 16, abrufbar unter: https://www.bmi.bund.de [Zugriff am 14.1.2022]; zu den gemischten Reaktionen im Allg. (,Unsicherheitsstrategie“; Regierung ,,als größtes Sicherheitsrisiko für Deutschland“) s. heise-online v. 9.9.2021.

2) Im Koalitionsvertrag der SPD/GRÜNE/FDP v. 24.11.2021 ist die „IT-Sicherheit“ u.a. mit einem Recht auf Verschlüsselung, einem Schwachstellenmanagement und einer Herstellerverantwortlichkeit für Sicherheitslücken in Produkten angesprochen (S. 16) und wird in einigen Punkten etwas anders akzentuiert als in der o.g. Cybersicherheitsstrategie (z. B. bzgl. Hackbacks, Verschlüsselung).

3) BMI, Cybersicherheitsstrategie für Deutschland 2021, S. 16

4) BMI, Cybersicherheitsstrategie für Deutschland 2021, S. 44, 50, $63 \mathrm{f} ., 77$.

5) Vgl. BT-Dr. 19/20708, S. 167

6) BMI, Cybersicherheitsstrategie für Deutschland 2021, S. 6, $12 \mathrm{ff}$.

7) Für den Bewertungszeitraum Juni 2020 bis Mai 2021 s. BSI, Die Lage der IT-Sicherheit in Deutschland 2021, S. 9.

8) Vgl. BSI, Die Lage der IT-Sicherheit in Deutschland 2021, S. 4.

9) $\mathrm{Zu}$ verschiedenen Begehungsformen von Cyberangriffen s. im Überblick auch Grzesiek, GuP 2021, 171, 172.

10) Zu zunehmenden Cyberattacken und Sicherheitslücken in Arztpraxen s. BT-Dr. 19/13438, S. 48; ärzteblatt.de v. 9.11.2021, abrufbar unter: https://www.aerzteblatt.de/nachrichten/128890/ Hacker-bedrohen-zunehmend-die-IT-in-Arztpraxen [Zugriff am 14. 1.2022].; mit Bsp. s.a. Grzesiek, GuP 2021, 171 m.w. N.

11) So unter Berufung auf das BSI Grätzel von Grätz, EHealth.COM v. 27. 10.2021, abrufbar unter: https://e-health-com.de/detailsnews/neuer-bsi-bericht-it-sicherheit-angespannt-bis-kritisch/ [Zugriff am 14.1.2022]

12) BKA, Cybercrime, Bundeslagebild 2019, S. 16f., $23 \mathrm{f}$.

13) heise online v. 17.9.2020; Grzesiek, GuP 2021, 171; vgl. BSI, Die Lage der IT-Sicherheit in Deutschland 2021, S. 15.

14) Angriffe gegen Krankenhäuser sollen im Jahr 2020 innerhalb von zwei Monaten um 45 Prozent gestiegen sein, e-health-com.de v. 15.7.2021, abrufbar unter: https://e-health-com.de/detailsnews/hackerangriffe-auf-krankenhaeuser-waehrend-coronagestiegen/ [Zugriff am 14.1.2022].

15) Vgl. BSI, Die Lage der IT-Sicherheit in Deutschland 2021, S. 39, 57.

16) BReg., BT-Dr. 19/24247, S. 3

17) www.healthcare-computing.de v. 9.11.2021, abrufbar unter: https://www.healthcare-computing.de/hacker-attacke-auf-medizin-dienstleister-medatixx-a-1073742/?xing_share=news; ärzteblatt.de v. 9. 11.2021, abrufbar unter: https://www.aerzteblatt.de/ nachrichten/128901/Cyberattacke-auf-Medatixx-Aerzte-sollenPasswoerter-aendern; heise-online v. 21.12.2021, abrufbar unter: https://heise.de/-6301881 [alle Zugriff am 14.1.2022]. 
lich vorsorglich ihre Passwörter für u.a. Server, Router, Firewalls oder die der TI-Konnektoren zu ändern, weil nicht ausgeschlossen werden konnte, dass beim Dienstleister gespeicherte Daten entwendet worden seien. Die Fälle zeigen, dass Cybersicherheit ebenso in Auftragsverhältnissen oder gar Auftragsketten mittelbar von großer Bedeutung sein kann ${ }^{18}$.

Angriffsziele sind ferner staatliche Einrichtungen, wie der DDoS-Angriff auf das COVID-19-Impfportal des Bundeslands Thüringen ${ }^{19}$ oder eine Ransomware-Attacke auf das irische Gesundheitssystem im Jahr $2020^{20}$ belegen. Auch andere Institutionen des Gesundheitswesens, wie z.B. die Bayrische Krankenhausgesellschaft, waren schon Ziel von „Hackerangriffen“"21. Für Aufsehen gesorgt hat ferner ein Cyber-Angriff auf die Europäische Arzneimittelagentur (EMA), bei dem Dokumente von Pharmaunternehmen, insbesondere Antragsunterlagen für die Zulassung eines Impfstoffs gegen das Coronavirus SARS-CoV-2 sowie interne, vertrauliche E-Mail-Korrespondenz, erlangt und anschließend ,geleakt“ werden konnten ${ }^{22}$.

Zudem entstehen Gefährdungen durch Fehlkonfigurationen, unterbliebene Updates und Sicherheitslücken in Software (wie zuletzt die Log4j-Schwachstelle Log4Shell) sowie wegen Fehlverhaltens, Unachtsamkeit und Bedienungsfehlern des Personals ${ }^{23}$ bzw. der Anwender. So waren Arztpraxen von Datenleaks betroffen ${ }^{24}$ und es traten Fälle auf, bei denen 30.000 Patientenakten und weitere Dokumente sowie radiologische Bilddaten von sog. PACS-Servern abrufbar waren $^{25}$. Ungeachtet dessen sollen im Jahr 2021 im Internet weltweit mehr als 45 Millionen medizinische Bilddateien darunter Röntgen-, CT- und MRT-Scans - auf ungeschützten Servern für jedermann frei zugänglich gewesen $\operatorname{sein}^{26}$.

Treiber für Gefahrenlagen im Gesundheitswesen sind die fortschreitende Digitalisierung und Technisierung vieler Prozesse. Die damit einhergehende Abhängigkeit von IT-Systemen wächst stetig und ein Ausfall kann große finanzielle Schäden für die Einrichtungen sowie gravierende Folgen für Rechtsgüter ihrer Patienten haben. Wie das BSI in seinem Lagebericht feststellt, kann insbesondere ein Verlust von Gesundheitsdaten möglicherweise lebenslange Folgen für Patienten nach sich ziehen ${ }^{27}$. Unabhängig von einer Verarbeitung personenbezogener Gesundheitsdaten ist Cybersicherheit im Gesundheitswesen also eine bedeutsame Voraussetzung der Sicherheit der Patienten- und Gesundheitsversorgung, weil sie auf die Funktionsfähigkeit der jeweiligen Einrichtung insgesamt abzielt.

Relevante Einrichtungen des Gesundheitswesens müssen vor diesem Hintergrund eine hinreichende Cybersicherheit gewährleisten. Dazu gehören vor allem Kliniken, Hersteller von (Medizin-)Produkten, Digitaler Gesundheitsanwendungen (DiGA), Arzneimittel- und Impfstoffhersteller, Forschungseinrichtungen, Arztpraxen, Medizinische Versorgungszentren (MVZ) und Versicherungen. Kritische Bereiche in diesen Einrichtungen sind u.a. Krankenhausinformationssysteme (KIS), Laborinformationssysteme (LIS), Radiologieinformationssysteme (RIS), Picture Archive and Communication Systeme (PACS) und Dokumenten-Management-Systeme (z.B. die elektronische Patientenakte). Bedeutsam ist Cybersicherheit überdies für innovative, auf IuK-Technik gestützte Versorgungsformen (z.B. Telemedizin) oder für die Medizintechnik und Medizinprodukte. Insbesondere Sicherheitsschwachstellen in Medizinprodukten können verheerende Folgen haben, z. B. könnte die Insulinpumpe eines Diabetikers ,gehackt" und manipuliert werden.

\section{Einordnung der Cybersicherheit und Bedeutung im Gesundheitswesen}

Im Kontext der Cybersicherheit werden unterschiedlichste, teils kontrovers diskutierte Begriffe verwendet ${ }^{28}$ : IT-Sicherheit, Datensicherheit, Informationssicherheit und Cybersi- cherheit. ,Sicherheit“ bedeutet im technischen Sinne jeweils die „Freiheit von unvertretbarem Risiko" ${ }^{29}$. IT-Sicherheit bezweckt die Sicherheit speziell von IT-Systemen; vor allem gegen Angriffe von außen. Das umfasst den Schutz von Daten in Rechner- und Informationssystemen vor Verlust oder Zerstörung, die durch vorsätzliche oder nicht vorsätzliche Handlungen durch nicht autorisierte Personen verursacht werden. Datensicherheit hingegen umfasst die Absicherung persönlicher Informationen gegen unbefugte Offenlegungen, Übermittlungen, Veränderungen und/oder Zerstörungen sowohl vorsätzlicher als auch fahrlässiger Art, und ist damit „untrennbarer Bestandteil“ des Datenschutzes. Informationssicherheit wiederum befasst sich mit der Sicherheit von in Daten enthaltenen Informationen vor unbefugten Offenlegungen, Übermittlungen, Veränderungen und/ oder Zerstörungen. Sie zielt damit auf Aufrechterhaltung der Vertraulichkeit, Integrität und Verfügbarkeit von Information unabhängig davon, ob die in den Daten enthaltenen Informationen einen Personenbezug aufweisen und unabhängig davon, ob die Informationen digital bzw. online/ offline verarbeitet werden. Sie bezieht sich damit zugleich auf in Papierform übertragene Informationen. Cybersicherheit (,cyber security“) versteht sich vor diesem Hintergrund als genereller Oberbegriff und umfasst alle Themenfelder sowohl der IT-Sicherheit, der Informationssicherheit, der Datensicherheit als auch des Datenschutzes ${ }^{30}$. Sie befasst sich generell mit der Sicherheit von Datenverarbeitungsvorgängen, insbesondere im digitalen und vernetzten Raum.

Wie erwähnt ist der Datenschutz ein bedeutsamer Aspekt der Cybersicherheit, aber gleichwohl von ihr abzugrenzen: Das Datenschutzrecht betrifft Voraussetzungen für Gewährleistung der informationellen Selbstbestimmung, vor allem mit Blick auf legitimierende Rechtsgrundlagen, Transparenz etc. Es besteht eine enge Verzahnung, denn ein ,angemessenes Datenschutzniveau“ im Sinne informationeller Selbstbestimmung setzt voraus, dass technischorganisatorische Maßnahmen getroffen werden (s. insb. Art. 32 DSGVO). Cybersicherheit und Datenschutz gehen daher „Hand in Hand“31.

18) Cybersicherheit dürfte daher auch ein wichtiger Faktor bei der Vertragsgestaltung mit Dienstleistern (und ggf. deren Sub-Dienstleistern) sein, der über die Gestaltung von Auftragsverarbeitungsvereinbarungen (Art. 28 Abs. 1, Abs. 3 S. 2 insb. lit. c sowie Abs. 4 DSGVO) hinausgeht, weil nicht nur die Sicherheit von Daten, sondern auch die Funktionsfähigkeit der IT-Systeme und ihrer Komponenten (Server, Router oder TI-Konnektoren) betroffen sein kann.

19) Vgl. BSI, Die Lage der IT-Sicherheit in Deutschland 2021, S. 39

20) ärzteblatt.de v. 14.5.2021; BSI, Die Lage der IT-Sicherheit in Deutschland 2021, S. 39.

21) Hierbei war E-Mail-Server der BKG mit einer Schadsoftware infiziert worden, s. heise online v. 2.12.2021, abrufbar unter: https://heise.de/-6281905 [Zugriff am 14.1.2022]

22) Vgl. BSI, Die Lage der IT-Sicherheit in Deutschland 2021, S. 10 39.

23) Zu den endogenen Ursachen s. Dittrich, GuP 2021, 165, 167 m. w. N.; Grzesiek, GuP 2021, 171, 176; Staufer, DÄB1. 2020, Heft 40, S. 2.

24) BSI, Die Lage der IT-Sicherheit in Deutschland 2020, S. 20.

25) Näher c't 25/2019, S. 16, s. heise online v. 22.11.2019, heise.de/4590103 [Zugriff am: 14.1.2022]; vgl. auch BayLDA, 9. Tätigkeitsbericht 2019, S. 66.

26) Analystenteam von CybelAngel v. 12.1.2021, datensicherheit.de, 12.1.2021, abrufbar unter: https://www.datensicherheit.de/schutzlosigkeit-online-zugriff-45-millionen-medizinische-bilder-welt [Zugriff am: 14.1.2022].

27) Vgl. BSI, Die Lage der IT-Sicherheit in Deutschland 2021, S. 26.

28) Instruktiv und vgl. zu den nachfolgenden Definitionen Kipker, in: Kipker, Cybersecurity, 1. Aufl. 2020, Kap. 1, Rdnr. 4; s. ferner auch Kammerloher, DuD 2021, 649.

29) Kipker, in: Kipker, Cybersecurity, 1. Aufl. 2020, Kap. 1, Rdnr. 3.

30) Zum Teil a.A. Kammerloher, DuD 2021, 649.

31) Vgl. treffend Kipker, in: Kipker, Cybersecurity, 1. Aufl, 2020, Kap. 1, Rdnr. 4. 
Enge Verbindungen des Cybersicherheitsrechts im Gesundheitswesen bestehen zudem mit den bedeutsamen Vertraulichkeitserwartungen aufgrund bestehender Berufsgeheimnisverpflichtungen, wie der ärztlichen Schweigepflicht ${ }^{32}$. Vertrauen zählt mit dem BVerfG zu den „Grundvoraussetzungen ärztlichen Wirkens, weil es die Chancen der Heilung vergrößert und damit - im Ganzen gesehen - der Aufrechterhaltung einer leistungsfähigen Gesundheitsfürsorge

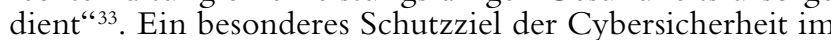
Gesundheitswesen ist daher die Bewahrung der Vertraulichkeit vor einer unbefugten Offenlegung oder einem unbefugten Zugang zu Patientengeheimnissen.

Besonders schädlich sind im Bereich der Gesundheitsversorgung außerdem eine mangelnde Erreichbarkeit, eine Vernichtung, ein Verlust oder eine Manipulation oder Verfälschung von Informationen. Ein weiteres wichtiges Schutzziel ist insoweit die Sicherstellung von Verfügbarkeit und Integrität der Informationen ${ }^{34}$. Im Kontext der Cybersicherheit ist dabei auch die Verfügbarkeit der IT-Systeme von Bedeutung. Wegen der Angewiesenheit auf den verlässlichen Einsatz von IuKTechnologien im Rahmen der Patientenversorgung ist Cybersicherheit also zugleich eine Voraussetzung für die individuelle Patientensicherheit und Sicherheit der Gesundheitsversorgung im Gemeinwesen. Ein Ausfall oder eine Unterbrechung der Versorgung kann zur Gefährdung von wichtigen Rechtsgütern wie Gesundheit und Leben führen. Sie ist damit nicht nur Grundvoraussetzung einer jeden Datenverarbeitung, sondern ebenso Voraussetzung für Vertrauen in digitale Techniken, die im Gesundheitswesen zunehmend Verbreitung finden sollen.

\section{Rechtsquellen der Cybersicherheit im Gesundheitswesen im Überblick}

Das Cybersicherheitsrecht im Gesundheitswesen ist nicht einheitlich kodifiziert. Es findet sich zumeist im Zusammenhang mit datenschutzrechtlichen Vorschriften normiert, sofern personenbezogene Daten Schutzgegenstand sind. Das hat zur Folge, dass mehrere, verstreut gelegene Rechtsquellen zu betrachten sind. Einschlägig sind vor allem die DSGVO und das BDSG (Art. 5 Abs. 1 lit. f, Art. 24, Art. 25 und Art. 32 DSGVO; $\$ 22$ Abs. 2 BDSG). Unter dem Blickwinkel der Datensicherheit enthält die DSGVO den allgemeinen, auch in allen Sektoren und Bereichen des Gesundheitswesens, zu beachtenden Rahmen, wobei geeignete technische und organisatorische Maßnahmen zum Schutz von Gesundheitsdaten im Fokus stehen, die ein dem Risiko angemessenes Schutzniveau gewährleisten. Ein defizitärer Schutz kann für die Verantwortlichen zu Bußgeldern (Art. 82 DSGVO) oder Schadensersatzforderungen von den Betroffenen (Art. 82 DSGVO) führen.

Ein besonderer Schutzbedarf besteht regelmäßig für $\mathrm{Ge}-$ sundheitsdaten i.S.v. Art. 9 DSGVO, da konkrete Verarbeitungskontexte oftmals besonders sensibel sind. Diese Sichtweise entspricht dem ,risikobasierten Ansatz“ der DSGVO, aber auch im Standard-Datenschutzmodell (SDM) ist für Gesundheitsdaten die Schutzbedarfskategorie „hoch“ vorgesehen ${ }^{35}$. Art. 9 Abs. 2 DSGVO betont vereinzelt „Bedingungen und Garantien“, ,,angemessene und spezifische Maßnahmen“ oder ,geeignete Garantien“ zur Wahrung der (Grund-)rechte der von der Datenverarbeitung betroffenen Personen. Gemeint sind damit prozedurale, technische und organisatorische Maßnahmen, also ebenfalls Maßnahmen der IT-, Informations- und Datensicherheit ${ }^{36}$.

Das Ziel von ,technischen und organisatorischen Maßnahmen" (vgl. Art. 32 DSGVO) ist die Gewährleistung von Schutzzielen (auch Gewährleistungs- oder Sicherheitsziele) der Informations- und Datensicherheit ${ }^{37}$. Sie sind der „,normative Anker“ für die Auswahl und Bestimmung von Schutzmaßnahmen gegen Risiken ${ }^{38}$. Eine explizite Anforderung zur Gewährleistung des Vertraulichkeitsschutz, als herauszuhebendem Schutzziel der Informationssicherheit im Gesundheitswesen, enthält Art. 9 Abs. 3 DSGVO, wonach die Datenverarbeitung nur durch Berufsgeheimnisträger oder Geheimhaltungsverpflichtete erfolgen darf ${ }^{39}$. Der Schutz des Patientengeheimnisses ist insoweit eine typische Schutzmaßnahme für Gesundheitsdaten ${ }^{40}$.

Cybersicherheit fokussiert nicht allein (personenbezogene) Daten, sodass das Datenschutzrecht keinen abschlieBenden rechtlichen Rahmen bietet. Spezifische, über den Aspekt der Datensicherheit hinausgehende, Regelungen zur Cybersicherheit sind vereinzelt in den Sozialgesetzbüchern enthalten (z.B. $\$ 75 b, \$ 75$ c oder $\$ \$ 306$ ff. SGB V). Darüber hinaus sind rechtliche Anforderungen in (untergesetzlichen) Vereinbarungen und Richtlinien der Selbstverwaltungspartner formuliert (z. B. zur Videosprechstunde ${ }^{41}$ und für Telekonsile). Ferner sind weitere bereichsspezifische Rechtsvorschriften zu beachten (z. B. im ImplantateregisterG). Außerdem haben strafrechtliche Bestimmungen Bedeutung für die Cybersicherheit (\$\$202a ff. u. $\$ \$ 303$ a f. StGB; $\$ 42$ BDSG) und das (ärztliche) Berufsrecht scheint Rücksicht darauf zu nehmen (vgl. \$10 Abs. 5 MBO- ̈̈).

Die Anforderungen der Cybersicherheit ergeben sich trotz der Fülle normativer Anknüpfungspunkte nicht konkret aus der DSGVO oder einer der einschlägigen Rechtsquellen. Zumeist erfolgt dort lediglich ein Verweis auf den unbestimmten Rechtsbegriff „Stand der Technik“"42. Hierüber erlagen technische Normen und Standards im Cybersicherheitsrecht Bedeutung, wie z.B. die Normenreihe ISO/IEC 2700x oder Standards des Bundesamts für Sicherheit in der Informationstechnik (BSI) mit seinen Mindeststandards ${ }^{43}$ oder dem IT-Grundschutz-Anforderungskatalog. Daneben sind unter Umständen branchenspezifische Sicherheitsstandards (B3S) ${ }^{44}$ einschlägig. Diese etablierten Methoden und „Best-Practices“ sind praktische Basis für die Implementierung von Cybersicherheit durch IT-Verantwortliche oder externe Fachdienstleister ${ }^{45}$.

Cybersicherheitsrecht wird zudem über den Aspekt der „Qualitätssicherung“ vermittelt, denn ein einrichtungsinternes Qualitätsmanagement umfasst zugleich den Aspekt der Daten- und Informationssicherheit ${ }^{46}$. Ferner ist sie -

32) Näher Dochow, MedR 2019, $279 \mathrm{ff}$.

33) BVerfGE 32, 373, 380

34) Weitere (gleichwertige) Schutzziele sind z.B. die Authentizität, Verbindlichkeit, Zurechenbarkeit, Nichtverkettung, Intervenierbarkeit und Transparenz. Vgl. zu den Schutzzielen Art. 5 Abs. 1 lit. f DSGVO; $\$ 75$ b SGB V sowie zu deren Bedeutung im Gesundheitswesen Dochow/Herpers/Raptis, in: Kipker, Cybersecurity, 2. Aufl. 2022 (erscheint voraussichtlich im 1. Halbjahr 2022), Kap. 16, Rdnrn. 5 ff.; Rost, DuD 2018, 13 ff.; Dochow, Telematik im Gesundheitswesen, 2017, S. 789 ff. m. w. N.

35) DSK, Standard-Datenschutzmodell, Version 2.0b v. 17.4.2020.

36) Weichert, in: Kühling/Buchner, DS-GVO, BDSG, 3. Aufl. 2020 , Art. 9, Rdnrn. $132 \mathrm{ff}$.

37) Es bestehen Überschneidungen von Datenschutz und Cyberbzw. Informationssicherheit, was deutlich wird, da zahlreiche Schutzziele des Datenschutzes auch für die Cyber- und Informationssicherheit von Bedeutung sind, vgl. BMI, Cybersicherheitsstrategie für Deutschland 2021, S. 11.

38) Vgl. Rost, DuD 2018, 1, 13

39) Näher Dochow, GesR 2016, 401, 405; Dochow, in: Dochow/Dörfer et al., Datenschutz in der Arztpraxis, 2019, S. 42.

40) S. o. bei II

41) S. dazu V., 3.

42) S.a. den Begriff „Regeln der Technik“

43) $₫ 8$ Abs. 1 S. 1 BSIG

44) Im Krankenhausbereich s. DKG, Branchenspezifischer Sicherheitsstandard für die Gesundheitsversorgung im Krankenhaus, Version 1.1 v. 22.10.2019; s. dazu auch Dittrich/Ippach, GesR 2021, 285, 287

45) Vgl. Kammerloher, DuD 2021, 649, 652

46) Vgl. G-BA, Qualitätsmanagement-Richtlinie/QM-RL v. 17.9. 2020, BAnz. AT 8.12.2020 B2; überzeugend herausgearbeitet im Hinblick auf $\$ 75$ c SGB V von Dittrich, GuP 2021, 165, 169 f. 
ebenso wie Cyber-Resilienz ${ }^{47}$ - unter dem Blickwinkel eines Betriebskontinuitäts-Managements zum Schutz vor betriebseinschränkenden Vorfällen ${ }^{48}$ als Geschäftsführungsaufgabe ${ }^{49}$ und im Rahmen von Sicherheitsobliegenheiten im Versicherungsbereich ${ }^{50}$ von zunehmender Bedeutung.

\section{Cybersicherheitsrecht in den Sektoren des Gesundheitswesens}

\section{Stationärer Sektor}

Im stationären Sektor muss zwischen den Einrichtungen unterschieden werden, die aufgrund einer bestimmten Größe und Relevanz für die Gesundheitsversorgung zu den Kritischen Infrastrukturen (KRITIS) gehören, und kleineren Einrichtungen, die freilich keine geringere Bedeutung für eine funktionierende Krankenhausversorgung haben. Für Kritische Infrastrukturen sind neben den Anforderungen der DSGVO $^{51}$ insbesondere die Regelungen des BSIG $^{52}$ (dazu a.) und für andere Einrichtungen sind im Geltungsbereich des SGB bestimmte Spezialvorschriften (dazu b.) einschlägig.

\section{a) Kritische Infrastrukturen (KRITIS) und Pflichten} nach dem BSIG

Für Großkrankenhäuser, die regelmäßig zu den Kritischen Infrastrukturen zählen, sind die Bestimmungen des BSIG bedeutsam. Kritische Infrastrukturen sind Organisationen oder Einrichtungen mit wichtiger Bedeutung für das staatliche Gemeinwesen, bei deren Ausfall oder Beeinträchtigung nachhaltig wirkende Versorgungsengpässe, erhebliche Störungen der öffentlichen Sicherheit oder andere dramatische Folgen eintreten würden ${ }^{53}$. Sie werden nach Sektoren und Branchen unterteilt und durch die BSI-KritisV ${ }^{54}$ festgelegt. Dem Sektor „Gesundheit“ unterfallen gem. $\$ 6$ Abs. 1 BSI-KritisV als KRITIS neben größeren Krankenhäusern im Übrigen auch Hersteller von Arzneimitteln und Medizinprodukten, Labore und Apotheken, soweit für bestimmte kritische Dienstleistungen, meist mit lebenserhaltender Relevanz, soweit bestimmte Schwellenwerte überschritten werden ${ }^{55}$. Bemessungskriterien sind dabei z.B. Umsätze pro Jahr, hergestellte oder abgegebene Arzneipackungen oder die Anzahl von Aufträgen zur Laboriumsdiagnostik. Die Einrichtungen werden wegen ihrer Bedeutung für das Funktionieren des Gemeinwesens zu den KRITIS gezählt unabhängig davon, ob sie privatwirtschaftlich oder öffentlich-rechtlich getragen sind.

Krankenhäuser zählen gem. $\$ 2$ Abs. 10 BSIG i.V. mit $\$ 6$ BSI-KritisV erst ab 30.000 vollstationären Behandlungsfällen pro Jahr zu den KRITIS ${ }^{56}$. Wenn dieser Schwellenwert überschritten ist, bestehen für sie bestimmte Pflichten insbesondere nach $\$ 8 \mathrm{a}, \int 8 \mathrm{~b}$ BSIG. Danach sind IT-Systeme und IT-Prozesse durch angemessene organisatorische und technische Vorkehrungen nach dem ,Stand der Technik“ abzusichern ( $\$ 8$ a Abs. 1 BSIG), wobei zur Gewährleistung der Anforderungen branchenspezifische Sicherheitsstandards (B3S) von Betreibern von KRITIS und ihrer Branchenverbände genügen können ( $\$ 8 \mathrm{a}$ Abs. 2 BSIG), soweit deren Eignung vom BSI festgestellt wurde ( $₫ 8$ a Abs. 2 S. 2 BSIG). Das ist für den „B3S-Krankenhaus“"57 der DKG seit Ende des Jahres 2019 der Fall. Für die praktische Umsetzung bieten solche Branchenstandards den Vorteil, dass sie einerseits die, nur mit unbestimmten Rechtsbegriffen umschriebenen, gesetzlichen Anforderungen näher konkretisieren und andererseits eine zeitgerechte und flexible Anpassung der regelmäßig evaluierten „B3S“ an den „Stand der Technik“ erfolgen kann ${ }^{58}$.

Die Erfüllung der Anforderungen nach $\$ 8$ a Abs. 1 BSIG muss durch die Betreiber von KRITIS alle zwei Jahre nachgewiesen werden ( $(8 \mathrm{a}$ Abs. $3 \mathrm{BSIG})$. Für das BSI besteht zudem eine Prüfbefugnis ( $\$ 8$ a Abs. 4 BSIG). Ferner sind Meldepflichten bei Störungen zu beachten ( $\$ 8$ b Abs. 4 BSIG $)^{59}$. In diesem Kontext sind Betreiber von KRITIS auch dazu verpflichtet, eine Kontaktstelle zu benennen ( $\$ 8 \mathrm{~b}$ Abs. 4
BSIG), weil Störungen unverzüglich über diese Kontaktstelle an das BSI zu melden sind. Die Bußgeldvorschriften des $\$ 14$ BSIG sichern die vorstehenden Pflichten ab.

b) Einrichtungen unterhalb der KRITIS-Schwellen und Pflichten nach $\$ 75 c$ SGB V

$\mathrm{Zu}$ den KRITIS zählen Schätzungen zur Folge nur 5-10\% aller Krankenhäuser ${ }^{60}$. Für Einrichtungen unterhalb der KRITIS-Schwellen, also Krankenhäuser mit geringeren Fallzahlen, besteht aber ein nicht minder großes Bedrohungspotenzial ${ }^{61}$. Dies hat der Gesetzgeber erkannt und mit dem Patientendaten-Schutz-Gesetz (PDSG) ${ }^{62}$ im Jahr 2020 die neue Regelung des $\$ 75$ c SGB V geschaffen, welche der Verbesserung der IT-Sicherheit in Krankenhäusern dienen soll und sich erkennbar an $\$ 8$ a Abs. 1 BSIG anlehnt ${ }^{63}$. Seit dem 1.1.2022 sind Krankenhäuser verpflichtet, ,nach dem Stand der Technik angemessene organisatorische und technische Vorkehrungen zur Vermeidung von Störungen der Verfügbarkeit, Integrität und Vertraulichkeit sowie der weiteren Sicherheitsziele ihrer informationstechnischen Systeme, Komponenten oder Prozesse zu treffen, die für die Funktionsfähigkeit des jeweiligen Krankenhauses und die Sicherheit der verarbeiteten Patienteninformationen maßgeblich sind."

Adressaten der Vorschrift sind alle Krankenhäuser, soweit sie nicht ohnehin als Betreiber Kritischer Infrastrukturen nach KRITIS verpflichtet sind ( $(75$ c Abs. 3 SGB V). Weil

47) Dies bedeutet, dass zentrale Prozesse in Gesundheitseinrichtungen auch unter außergewöhnlichen Umständen, wie Sicherheitsvorfällen, funktionsfähig bzw. im Ernstfall schnell wiederherstellbar sein müssen (vgl. Kammerloher, DuD 2021, 649, 650 f.; s. a. Dittrich/Dochow/Ippach, GesR 2021, 613, 624 zum Entwurf einer Resilienz-Richtlinie der EU). Cyber-Resilienz steht damit in engem Zusammenhang mit der Cybersicherheit.

48) Dazu s.a. Dittrich/Dochow/Ippach, GesR 2021, 613, 624f. bzgl eines Entwurfs einer Resilienz-Richtlinie der EU.

49) Näher Schmidt-Versteyl, NJW 2019, 1637 ff.

50) Zum Risikotransfer durch Cyber-Versicherungen s. Grzesiek, GuP 2021, 171, 177. Ausgangspunkt können auch hier Begriffe wie ,Sorgfaltspflichten“" sein.

51) S. o. III. Diese sind nur einschlägig bei einer Verarbeitung von personenbezogenen (Gesundheits-)Daten (Art. 2 DSGVO).

52) Gesetz über das Bundesamt für Sicherheit in der Informationstechnik (BSI-Gesetz - BSIG) v. 14.8.2009 (BGBl. I S. 2821), zul. geänd. Art. 12 des G. v. 23.6.2021 (BGB1. I S. 1982).

53) S. https://www.kritis.bund.de/SubSites/Kritis/DE/Einfuehrung/ einfuehrung_node.html [Zugriff am 14.1.2022]; s. jüngst auch Mester, DuD 2021, 627 m. w. N., näher zum Ganzen Beucher/Fromageau, in: Kipker, Cybersecurity, 1. Aufl. 2020, Kap. 12, Rdnrn. 1, 11, $26 \mathrm{ff}$

54) Verordnung zur Bestimmung Kritischer Infrastrukturen nach dem BSI-Gesetz (BSI-Kritisverordnung - BSI-KritisV) v. 22. 4. 2016 (BGB1. I S. 958), zul. geänd. durch Artikel 1 der VO v. 6.9.2021 (BGBl. I S. 4163)

55) S. Anhang 5, Teil 3 der BSI-KritisV. Ferner zu nennen sind bspw. Private Krankenversicherungen mit 2.000.000 Leistungsfällen und gesetzliche Kranken- und Pflegeversicherungen mit mehr als 3.000.000 Versicherten. Zur Telematikinfrastruktur s. u. VI.

56) S. Anhang 5, Teil 1 Nr. 1 Ziff. 1.1, Teil 3, Nr. 1 Ziff. 1.1 der BSIKritisV.

57) DKG, Branchenspezifischer Sicherheitsstandard für die Gesundheitsversorgung im Krankenhaus, Version 1.1 v. 22.10.2019.

58) Vgl. auch näher zu den Inhalten des B3S-Krankenhaus Dittrich, GuP 2021, 165, 167f.; vgl. auch Hesral, in: Schlegel/Voelzke, jurisPK-SGB V, 4. Aufl. (Stand: 11. 5. 2021), \$75c SGB V, Rdnr. 14.

59) Näher zum Ganzen Beucher/Fromageau, in: Kipker, Cybersecurity, 1. Aufl. 2020, Kap. 12, Rdnrn. 68 ff.

60) Jorzig/Sarangi, Digitalisierung im Gesundheitswesen, 2020, S. 85

61) S. BT-Dr. 19/20708, S. 167; näher Becker/Gärtner, KH 2021, 292, $293 \mathrm{ff}$

62) Gesetz zum Schutz elektronischer Patientendaten in der Telematikinfrastruktur (Patientendaten-Schutz-Gesetz - PDSG) v. 14. 10.2020 (BGBl. I S. 2115).

63) Zu den Unterschieden und krit. zur Frage des ,Mehrwerts“ Dittrich, GuP 2021, 165, 167 ff.; krit. auch Becker/Gärtner, KH 2021, 292, $293 \mathrm{ff}$. 
der Bezugspunkt „Krankenhaus“ gewählt wurde, ist die stationäre und - anders als nach der BSI-KritisV ${ }^{64}$ - neben der teilstationären auch die ambulante Krankenhausversorgung eingeschlossen.

Durch die Verpflichteten sind Maßnahmen bei IT-Systemen, Prozessen und Komponenten mit dem Ziel der Sicherheit der verarbeiteten Patienteninformationen aber vor allem des Erhalts der Funktionsfähigkeit des jeweiligen Krankenhauses zu ergreifen. Das bedeutet, dass - anders als nach Art. 32 DSGVO - auch datenschutzrechtlich irrelevante oder rein technische Daten sowie IT-Systeme, Prozesse, Komponenten zum Schutzgegenstand zählen. Bei der Implementierung von Maßnahmen gilt indes der Verhältnismäßigkeitsgrundsatz, denn es sind nur ,,angemessene" Maßnahmen notwendig. Das ist gem. \$75c Abs. 1 S. 2 SGB V der Fall, wenn der erforderliche Aufwand nicht außer Verhältnis zu den Folgen eines Ausfalls oder einer Beeinträchtigung des Krankenhauses oder der Sicherheit der verarbeiteten Patienteninformationen steht.

Nach $\$ 75$ c Abs. 2 SGB V genügen branchenspezifische Sicherheitsstandards (B3S) den Anforderungen, soweit deren Eignung gem. $\int 8$ a Abs. 2 S. 2 BSIG vom BSI festgestellt wurde. Der „B3S-Krankenhaus“65 ist demnach auch für Kliniken, die nicht zu den KRITIS zählen, eine Orientierungshilfe zur Einhaltung des Standes der Technik.

Die Erfüllung der Pflichten, die gem. $₫ 75$ c Abs. 1 S. 3 SGB V alle zwei Jahre eine Anpassung an den aktuellen Stand der Technik erfordert, ist weder nachzuweisen ${ }^{66}$ noch durch eine Bußgeldvorschrift abgesichert ${ }^{67}$. Insoweit wird im Bereich der Cybersicherheit hier (noch) nicht auf Sanktionen ${ }^{68}$, sondern - seit dem KHZG ${ }^{69}$ - vielmehr auf Förderungen und Anreize gesetzt ${ }^{70}$

\section{Ambulanter Sektor}

a) Richtlinie zur IT-Sicherheit für Leistungserbringer nach $\$ 75 b$ SGB V

Aufgrund zunehmender Cyberattacken und Sicherheitslücken in (Zahn-)Arztpraxen ${ }^{71}$ wurde mit dem DVG ${ }^{72}$ im Jahr 2019 die neue Aufgabe der Kassenärztlichen Bundesvereinigungen (KBV und KZBV) in das SGB V aufgenommen, Vorgaben für die Gewährleistung von IT-Sicherheit in der vertragsärztlichen und vertragszahnärztlichen Versorgung in einer Richtlinie festzulegen $(\$ 75 b$ SGB V) und diese jährlich an den Stand der Technik und das Gefährdungspotential anzupassen $(\$ 75 b \text { Abs. } 3 \text { S. } 1 \text { SGB V })^{73}$. Die Abhängigkeit von IT-Systemen in der vertrags(zahn)ärztlichen Versorgung und das wachsende Bedrohungspotenzial durch zunehmend zielgerichtete, technologisch ausgereiftere und komplexere Angriffe lassen auch in (Zahn)Arztpraxen, die als Kleinstunternehmen oder kleine Unternehmen regelmäBig nicht dem Anwendungsbereich des BSIG unterfallen ${ }^{74}$ den verstärkten Bedarf zur Gewährleistung von IT-Sicherheit erkennen ${ }^{75}$. Der Schutzbedarf besteht dabei - unabhängig von einer Verarbeitung personenbezogener Daten - für die ,dort eingesetzten informationstechnischen Systeme“"76. Das betrifft in Arztpraxen also die Hardware (z. B. Server, Router, TI-Konnektoren) und die Software (z.B. Praxisverwaltungssoftware). Das Schutzziel von $\$ 75 \mathrm{~b}$ SGB V ist ein Doppeltes: Der Schutz von IT-Systemen und von personenbezogenen Daten ${ }^{77}$. Eine alleinige Bezugnahme auf das Datenschutzrecht steht demnach nicht im Einklang mit dem gesetzlichen Auftrag gem. \$75b SGB V, weshalb die Regelung auch nicht etwa im BDSG lokalisiert ist. Die in den Richtlinien zu konkretisierenden Vorgaben haben insoweit nicht allein den Zweck, technisch-organisatorische Maßnahmen im Interesse der Sicherheit personenbezogener Daten i.S. v. Art. 32 DSGVO zu standardisieren ${ }^{78}$.

In der Richtlinie sind konkrete Sicherheitsmaßnahmen aufzuführen, die von den Leistungserbringern umzusetzen sind. Adressaten im ambulanten Bereich sind gem. $\$ 75 b$ Abs. 4
S. 1 SGB V demgemäß Vertrags(zahn)ärzte. Sie gelten gem. \75b Abs. 4 S. 2 SGB V aber auch für an der vertrags(zahn) ärztlichen Versorgung teilnehmende Krankenhäuser, die keine Maßnahmen nach $₫ 8$ a BSIG ergreifen oder umsetzen ${ }^{79}$. Die IT-Sicherheitsrichtlinien sind hingegen, formal nach dem SGB V betrachtet, nicht verbindlich für ausschließlich privatärztlich tätige Ärzte. Gleichwohl können die Richtlinien als Branchenstandard für diese Praxen eine Orientierung für geeignete Sicherheitsmaßnahmen nach dem Stand der Technik - auch gemäß den Vorgaben der DSGVO ${ }^{80}$ - bieten und sie daher zur Einhaltung der Anforderungen verpflichten.

Bei der Umsetzung der Maßnahmen sind nicht alle denkbaren technischen und finanziell unter Umständen für kleinere und Kleinunternehmen kaum darstellbare Möglichkeiten auszuschöpfen. Vielmehr ist in $\$ 75 \mathrm{~b}$ Abs. 2 SGB V ein Verhältnismäßigkeitsprinzip enthalten. Danach müssen die Anforderungen, die in den Richtlinien festzulegen sind, geeignet sein, ,abgestuft im Verhältnis zum Gefährdungspotential und dem Schutzbedarf der verarbeiteten Informationen, Störungen der informationstechnischen Systeme, Komponenten oder Prozesse" hinsichtlich der Schutzziele zu vermeiden. Der Schutzbedarf richtet sich damit nach dem Gefährdungspotential und die Maßnahmen sind risikoorientiert an u. a. den klassischen Schutzzielen auszurichten: Verfügbarkeit, Integrität und Vertraulichkeit.

Eine Nichtbeachtung der Richtlinien kann einen Verstoß gegen vertrags(zahn)ärztliche Pflichten darstellen, was unter Umständen vertrags(zahn)ärztliche Disziplinarmaßnahmen

64) Zur Bezugnahme auf lediglich die stationäre medizinische Versorgung s. $\$ 6$ Abs. 1 Nr. 1, Abs. 5 i.V. mit Anhang 5, Teil 3, Nr. 1, Ziff. 1.1 BSI-KritisV.

65) S. o. bei IV., 1., a).

66) Anders im KRITIS-Bereich: $\$ 8$ a Abs. 3 BSIG. Auch Meldepflichten wie nach $\$ 8$ b Abs. 4 BSIG bestehen nicht. Zu den Unterschieden im Detail s. Dittrich, GuP 2021, 165, $167 \mathrm{f}$.

67) Anders im KRITIS-Bereich: $\$ 8$ a Abs. 1 i.V. mit $\$ 14$ Abs. 2 Nr. 2 BSIG und $₫ 8$ a Abs. 3 i. V. mit $\$ 14$ Abs. 1 BSIG. Denkbar wäre eine Aufnahme einer Bußgeldbewehrung in $₫ 397$ SGB V gewesen.

68) Krit. Dittrich, GuP 2021, 165, $169 \mathrm{ff}$.

69) Gesetz für ein Zukunftsprogramm Krankenhäuser (Krankenhauszukunftsgesetz - KHZG) v. 23.10.2020 (BGBl. I S. 220); s. dazu Dillschneider/Gross, KH 2020, 976, 977 ff.

70) Krankenhauszukunftsfonds ( $\$ 14$ a KHG); s. dazu Dochow/Herpers/Raptis, in: Kipker, Cybersecurity, 2. Aufl. 2022 (erscheint voraussichtlich im 1. Halbjahr 2022), Kap. 16, Rdnr. 17.

71) S. die Bsp. bei Grzesiek, GuP 2021, 171 m.w. N.

72) Digitale-Versorgung-Gesetz (DVG) vom 9.12.2019 (BGBl. I S. 2562).

73) S.a. den Auftrag zur Erstellung einer Richtlinie zur Zertifizierung von IT-Sicherheitsunternehmen ( $\$ 75 \mathrm{~b}$ Abs. 5 S. 1 SGB V), um die Leistungserbringer bei der Auswahl eines geeigneten Dienstleisters zu unterstützen; s. dazu Dochow/Herpers/Raptis, in: Kipker, Cybersecurity, 2. Aufl. 2022 (erscheint voraussichtlich im 1. Halbjahr 2022), Kap. 16, Rdnr. 186.

74) S. $\$ 8 d$ Abs. 1 BSIG.

75) Vgl. BT-Dr. 19/13438, S. 48.

76) BT-Dr. 19/13438, S. 48; vgl. auch Kircher, in: Becker/Kingreen, SGB V, 7. Aufl. 2020, $\$ 75$ b, Rdnr. 1.

77) Die Interessen bedingen sich gegenseitig. Zum engen Zusammenhang von Datenschutz und IT-Sicherheit bzw. Datensicherheit und technischen und organisatorischen Sicherheitsmaßnahmen s. Conrad et al., in: Auer-Reinsdorff/Conrad, Hdb. IT- und Datenschutzrecht, 3. Aufl. 2019, \$33, Rdnr. 180; Voskamp, in: Kipker, Cybersecurity, 1. Aufl. 2020, Kap. 5, Rdnrn. 2, 5 f.

78) So aber KBV, Richtlinie nach $\$ 75 b$ SGB V über die Anforderungen zur Gewährleistung der IT-Sicherheit, Stand: 22.1.2021, Präambel, s.a. ausdrücklich: „,erfüllen [...] Vorgaben der Datenschutz-Grundversordnung“"

79) Die rechtliche Verpflichtung allein dazu genügt nicht; die Maßnahmen nach BSIG sind tatsächlich umzusetzen; vgl. Kircher, in: Becker/Kingreen, SGB V, 7. Aufl. 2020, \$75b, Rdnr. 5.

80) Art. 32 DSGVO, s.o. bei III. sowie unten IV., 2., b). Für Grzesiek, GuP 2021, 171 folgt dies allein aufgrund der Bezugnahme auf Art. 32 DSGVO, was jedoch die bereitere Ausrichtung der IT- oder Cybersicherheit außer Acht ließe. 
gem. $₫ 81$ Abs. 5 SGB V nach sich zieht ${ }^{81}$. Daneben sind finanzielle Folgen gem. Art. 82, 83 DSGVO sowie strafrechtliche Konsequenzen gem. $\$ 203$ StGB in Einzelfällen denkbar, wenn eine hinreichende Sicherheit der verarbeiteten Gesundheitsdaten und Patientengeheimnisse nicht gewährleistet ist.

Die hier exemplarisch betrachtete Richtlinie der KBV ,über die Anforderungen zur Gewährleistung der zur ITSicherheit" (nachfolgend ,IT-Sicherheitsrichtline“) ${ }^{82}$ differenziert einerseits nach der Größe der Praxis und andererseits mit Blick auf den Einsatz medizinischer Großgeräte. Es sind mithin je nach Größe der Praxis unterschiedliche technische und organisatorische Anforderungen für konkrete Sicherheitsmaßnahmen definiert. Die Arztpraxen werden dabei in drei Kategorien eingeteilt: Kleine, mittelgroße und große Praxen. Maßgeblich für die Klassifizierung ist die Anzahl von Personen, die ständig mit der Datenverarbeitung betraut sind. In kleinen Praxen sind fünf, in mittleren Praxen sech bis 20 und großen Praxen mehr als 20 ,,datenverarbeitende“ Personen tätig. Großpraxen sollen außerdem solche Praxen sein, in denen die Datenverarbeitung über den ,normalen“ Umfang hinausgeht ${ }^{83}$, wobei „Groß-MVZ mit krankenhausähnlichen Strukturen" und Labore genannt werden.

Die Unterscheidung nach Praxisgrößen überzeugt nicht vollständig, weil die Größe der Praxis nicht notwendigerweise mit möglichen Cybersicherheits-Risiken korrelieren muss $^{84}$. Das Risiko hängt nicht allein von der Anzahl der Personen ab, die mit der Datenverarbeitung betraut sind, sondern vielmehr von der Art und vom Umfang der Daten und ihrer Verarbeitung sowie von den eingesetzten IT-Systemen und Anwendungen oder vom Grad der Vernetzung und Automatisierung ${ }^{85}$. Eine dementsprechende Abstufung der Anforderungen im Verhältnis zum Gefährdungspotential und dem Schutzbedarf wäre möglich gewesen (vgl. \$ $75 c$ Abs. 2 SGB V). Sachgemäß ist vor diesem Hintergrund die Abstufung im Hinblick auf den Einsatz von Großgeräten, weil dies mit höheren Risiken einhergehen kann.

Neben den allgemeinen Regelungen (Anwendungsbereich, Adressaten, Ziele, Praxisgrößen etc.), die zum Teil lediglich den Inhalt von $\$ 75 \mathrm{~b}$ SGB V wiederholen, definiert die IT-Sicherheitsrichtlinie in fünf Anlagen spezifische Anforderungen für einzelne „Zielobjekte“ nach den Kategorien „Software“ (Programme, Apps) und „Hard-

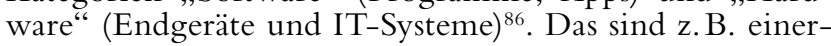
seits Mobile Apps, Internetanwendungen oder Office-Produkte und andererseits z. B. Tablets oder Smartphones. Die in Anlage 1 beschriebenen konkreten IT-Sicherheitsmaßnahmen sind von allen Praxen umzusetzen, die in Anlage 2 beschriebenen zusätzlich nur von den mittleren Praxen, die in Anlage 3 zusätzlich nur von großen Praxen und die in Anlage 4 zusätzlich von Praxen mit medizinischen Großgeräten wie z.B. CT, MRT. Die Anforderungen sind durch Maßnahmen vom Praxisinhaber umzusetzen, soweit die Zielobjekte in der Praxis genutzt werden.

Die Richtlinie umfasst in Anlage 5 schließlich Anforderungen an die sichere Installation und Wartung von dezentralen Komponenten und Diensten der Telematikinfrastruktur, die in der vertrags(zahn)ärztlichen Versorgung genutzt werden (s. $\$ 75 b$ Abs. 1 S. 2 SGB V). Diese Verpflichtung für alle Praxen, die mit der Telematikinfrastruktur verbunden sind, ist sachgemäß, weil Konnektoren oder Kartenlesegeräte regelmäßig wichtige Komponenten der IT-Systeme der Leistungserbringer sind.

Die Differenzierung nach ,soll“" und ,muss“-Vorgaben hat nur begrenzte Bedeutung, weil weder $\$ 75 \mathrm{~b}$ SGB V eine solche Abstufung vorsieht noch die DSGVO. Es ist vielmehr eine IT- bzw. Datensicherheit nach dem „Stand der Technik “ zu gewährleisten. Soweit in der IT-Sicherheitsrichtlinie der KBV dieser Standard abgebildet wird, gelten mithin diese Anforderungen als Maßstab, um die Anforderungen u.a. der DSGVO zu wahren, aber etwa auch um eine hinreichende Deckung bei etwaigen Cyberversicherungen durch
Erfüllung der Sicherheitsobliegenheiten ${ }^{87} \mathrm{zu}$ erlangen. Allerdings kann unter Berücksichtigung des zu erwartenden Risikos bei der Implementierung von Maßnahmen mit Blick auf das auch in $\$ 75$ b Abs. 2 SGB V akzentuierte Verhältnismäßigkeitsprinzip in einzelnen Fällen eine Abstufung des Pflichtengrades zulässig $\operatorname{sein}^{88}$. Eine holzschnittartige Vorgehensweise ist hierdurch indes nicht gedeckt.

Die Erfüllung der Anforderungen ist zeitlich gestaffelt. In einer ersten Stufe sind die Maßnahmen von den Verpflichtungsadressaten seit dem 1.4.2021 umzusetzen. Es handelt sich hierbei weitgehend um Basismaßnahmen ${ }^{89}$. Seit dem 1.1.2022 gilt die zweite Stufe der IT-Sicherheitsrichtlinie, welche für bestimmte Einsatzbereiche erweiterte Vorgaben zur IT-Sicherheit in Arztpraxen vorsieht. Das gilt z. B. für den Einsatz von Tablets und Smartphones. Für den Schutz der dezentralen Komponenten der Telematikinfrastruktur in der Umgebung der Arztpraxen ist insbesondere bei einem (grundsätzlich nicht empfohlenen) Parallelbetrieb des Konnektors neben einem anderen Zugang zum Internet ${ }^{90}$ auf zusätzliche Sicherheitsmaßnahmen zum Schutz des ITSystems in der Arztpraxis zu achten. In einer dritten Stufe $\mathrm{ab}$ dem 1.7.2022 haben mittlere und große Praxen weitere Anforderungen zu erfüllen, die das Mobile Device Management und die Netzwerksicherheit betreffen ${ }^{91}$, wie $z$. B. die Erstellung einer praxisinternen Richtlinie zur Benutzung von mobilen Endgeräten.

Insgesamt konkretisiert die IT-Sicherheitsrichtlinie der KBV nach eigenem Verständnis den „Stand der Technik“ im Sinne von Art. 32 DSGVO $^{92}$, aber in der Sache auch weiterer cybersicherheitsrechtlicher Vorschriften. Die Spezifizierung der Vorgaben dürfte für die praktische Umsetzung von IT-Sicherheitsmaßnahmen im Grundsatz hilfreich sein ${ }^{93}$. Wegen der jedenfalls für den Schutz von personenbezogenen Daten bereits langjährig bestehenden Verpflichtungen ${ }^{94}$, sind in der Richtlinie indes keine völlig ,neuen“ Pflichten aufgeführt ${ }^{95}$. Die IT-Sicherheitsrichtlinie bietet überdies lediglich ein Basiskonzept

81) Dazu Dittrich/Ippach, GesR 2021, 285, $288 \mathrm{ff}$.

82) KBV, Richtlinie nach $\$ 75$ b SGB V über die Anforderungen zur Gewährleistung der IT-Sicherheit, Stand: 22.1.2021; näher Dochow/Herpers/Raptis, in: Kipker, Cybersecurity, 2. Aufl. 2022 (erscheint voraussichtlich im 1. Halbjahr 2022), Kap. 16, Rdnrn. $179 \mathrm{ff}$.

83) A.A Grzesiek, GuP 2021, 171, der auf einen „,erheblichen“ Umfang der Datenverarbeitung abstellt.

84) Vgl. zur ähnlichen Problematik bei $\$ 38$ BDSG Dochow, in: Dochow/Dörfer et al., Datenschutz in der Arztpraxis, 2019, S. $124 \mathrm{ff}$. Dochow, PinG 2018, $53 \mathrm{ff}$.

85) Dochow/Herpers/Raptis, in: Kipker, Cybersecurity, 2. Aufl. 2022 (erscheint voraussichtlich im 1. Halbjahr 2022), Kap. 16, Rdnr. 232.

86) Näher Dochow/Herpers/Raptis, in: Kipker, Cybersecurity, 2. Aufl. 2022 (erscheint voraussichtlich im 1. Halbjahr 2022), Kap. 16 , Rdnr. 190 ff.; s.a. Grzesiek, GuP 2021, 171, 173 f.

87) Vgl. Grzesiek, GuP 2021, 171, 177.

88) Zu dem Gedanken s. Dochow/Herpers/Raptis, in: Kipker, Cybersecurity, 2. Aufl. 2022 (erscheint voraussichtlich im 1. Halbjahr 2022), Kap. 16, Rdnr. 182

89) Vgl. Dittrich/Ippach, GesR 2021, 285.

90) Vgl. zu Störungen BSI, Die Lage der IT-Sicherheit in Deutschland 2021, S. 52; s.a. zur Alternative der Reihenschaltung Dochow/Herpers/Raptis, in: Kipker, Cybersecurity, 2. Aufl. 2022 (erscheint voraussichtlich im 1. Halbjahr 2022), Kap. 16, Rdnr. 228.

91) Grzesiek, GuP 2021, 171, 174.

92) Präambel der Richtlinie, s. a. Dittrich/Ippach, GesR 2021, 285.

93) Weitere Hinweise zur Richtlinie finden sich hier: https://hub. kbv.de/display/itsrl [Zugriff am 14.1.2022]

94) Erinnert sei bereits von Inkrafttreten der DSGVO an die Anlage zu $₫ 9$ S. 1 BDSG a.F., deren Anforderungen zum Teil auch in der früheren „Technische Anlage“ zu den Hinweisen und Empfehlungen zur ärztlichen Schweigepflicht, Datenschutz und Datenverarbeitung in der Arztpraxis von BÄK und KBV konkretisiert waren.

95) A. A. wohl Grzesiek, GuP 2021, 171, 175: ,neue rechtliche Pflichten“. 
für ein „Mindestmaß“ an zu ergreifenden Maßnahmen für die IT-Sicherheit in Arztpraxen ${ }^{96}$. Sie ist allerdings $\mathrm{zu}$ stark auf die IT-Sicherheit fokussiert und deckt andere wichtige Aspekte der Cybersicherheit, wie eine physische, organisatorische und personelle Sicherheit, nicht genügend $\mathrm{ab}^{97}$. Um eine hinreichende Widerstandsfähigkeit (Resilienz) gegen Cyberangriffe zu erreichen, bedarf es weiterer Maßnahmen, wie die Erstellung eines ITSicherheitskonzeptes einschließlich einer Notfallstrategie und der Erstellung von internen Verhaltensrichtlinien für Beschäftigte $^{98}$.

\section{b) Einrichtungen außerhalb der Geltung des SGB}

Für ausschließlich privatärztlich tätige Inhaber von Arztpraxen oder Apotheken sowie Angehörige anderer Berufsgruppen im Gesundheitswesen, die nicht den Regelungen des SGB oder dem BSIG unterfallen, sind die Vorgaben der DSGVO und die in $\$ 22$ Abs. 2 BDSG nicht abschlieBend aufgeführten Maßnahmen zu ergreifen, die bei einer Verarbeitung von Gesundheitsdaten gelten. Dabei ist der Stand der Technik, Implementierungskosten sowie Art, Umfang, Umstände und Zwecke der Verarbeitung von Gesundheitsdaten sowie die unterschiedliche Eintrittswahrscheinlichkeit und Schwere der mit der Verarbeitung verbundenen Risiken für die Rechte und Freiheiten natürlicher Personen zu berücksichtigen. $\mathrm{Zu}$ den sehr allgemein gehaltenen Anforderungen des Katalogs zählt z. B. die Verschlüsselung personenbezogener Daten ${ }^{99}$. Ein Maßstab zur Einhaltung der rechtlichen Anforderungen nach dem Stand der Technik kann u. a. die IT-Sicherheitsrichtlinie der KBV bilden ${ }^{100}$.

\section{Cybersicherheitsrecht in besonderen Bereichen des Gesundheitswesens}

Wegen der übergreifenden Bedeutung von Informationstechnologie und digitalisierten Arbeitsweisen in einer immer stärker vernetzten Gesundheitsversorgung lässt sich die Cybersicherheit nicht mehr allein in den Grenzen der Sektoren betrachten. Daher sind besondere, vorliegend nicht abschließend aufgeführte Bereiche zu betrachten, für welche die allgemeinen Regelungen nach der DSGVO und des BDSG gelten, soweit personenbezogene Daten der Schutzgegenstand $\operatorname{sind}^{101}$. Für bestimmte kritische Dienstleistungen, z. B. für Hersteller von Arzneimitteln und Medizinprodukten, Labore und Apotheken, gelten darüber hinaus die oben erwähnten KRITIS-Vorgaben ${ }^{102}$.

Nachfolgend sollen indes lediglich die spezifischen Anforderungen in dem Bewusstsein skizziert werden, dass die vorgenannten rechtlichen Vorgaben ebenfalls zu berücksichtigen sind, soweit sie nicht durch spezialgesetzliche Bestimmungen vollständig verdrängt werden ${ }^{103}$. Spezifische Anforderungen gelten etwa im Bereich von Medizinprodukte (s. dazu nachfolgend 1.) oder für Digitale Gesundheitsanwendungen (s. dazu 2.) und - nur rudimentär ausgeprägt - im Bereich Telemedizin (s. dazu 3.). Daneben ist Cybersicherheit bei den hier nicht näher betrachteten Biobanken oder Register im Gesundheitswesen (z. B. Krebsregister, Transplantationsregister, Samenspenderregister, Implantateregister) ${ }^{104}$, im Rahmen des Melde- und Informationssystems im Bereich des Infektionsschutzes (DEMIS) sowie bei Cloud-Computing oder Online-Prozesse zur Terminorganisation sicherzustellen.

\section{Medizinprodukte}

Cybersicherheit kann auch bei Medizinprodukten ein bisweilen existenzielles Thema sein. Sicherheitsschwachstellen treten z.B. bei Insulinpumpen, Beatmungsgeräten oder Patientenmonitoren auf ${ }^{105}$. Fehlfunktionen oder Funktionsverluste infolge eines Cyberangriffs können hier lebensbedrohliche Folgen haben. Medizinprodukte i. S.v. Art. 2 Nr. 1 MDR sind neben Hardware auch Software oder KI-basierte Software für den medizinischen Bereich, soweit ein medizinischer Zweck gegeben ist. Zwar fallen Informationssysteme als solche nicht unter den Begriff des Medizinproduktes. Gleichwohl bestehen auch für diese Risiken bei der Einbindung von Medizinprodukten in die ITLandschaft von Einrichtungen im Gesundheitswesen und wegen Wechselwirkungen zwischen der Software und der IT-Umgebung. Cybersicherheit ist mithin auch hier ,,ganzheitlich" zu betrachten.

Für Medizinprodukte sind mehrere Regelungen einschlägig, je nachdem ob sie zu den KRITIS zählen oder nicht. Die Versorgung mit unmittelbar lebenserhaltenden Medizinprodukten unterfällt im Bereich Herstellung und Abgabe gem. $\$ 6$ Abs. 1 Nr. 2, Abs. 2 KritisV, sodass die bereits erwähnten Pflichten gelten ${ }^{106}$. Ansonsten sind die ebenfalls bereits erwähnten allgemeinen Anforderungen nach der DSGVO zu wahren, soweit eine Verarbeitung personenbezogener Daten, in der Regel Gesundheitsdaten gem. Art. 9 DSGVO, erfolgt ${ }^{107}$.

Spezifische Anforderungen ergeben sich aus dem Medizinprodukterecht ${ }^{108}$. Cybersicherheit als einer der grundlegenden Sicherheitsanforderungen ist Teil des Risikomanagements und damit Voraussetzung für das Inverkehrbringen und die Inbetriebnahme mit CE-Kennzeichnung ${ }^{109}$. Die Entwicklung und Herstellung nach dem Stand der Technik umfasst ausdrücklich Aspekte der Informationssicherheit (Art. 5 Abs. 2 i. V. mit Anhang I, Ziff. 17.2 MDR). Konkretisierungen dazu ergeben sich aus den einschlägigen technischen Normen (z. B. ISO 14971).

Für das Cybersicherheitsrecht von Bedeutung sind auch Meldepflichten bei mutmaßlich schwerwiegenden Vorkommnissen, wie z.B. Fehlfunktionen mit der Folge einer vorübergehenden oder dauerhaften schwerwiegenden Verschlechterung des Gesundheitszustands oder des Todes eines Patienten ( $\$ 5$ i. V. mit $\$ 2$ MPAMIV) sowie die Verpflichtung des Betreibers zu sicherheitstechnischen Kontrollen nach allgemein anerkannten Regeln der Technik (\$11 MPBetreibV).

96) Grzesiek, GuP 2021, 171, 175.

97) Näher Dochow/Herpers/Raptis, in: Kipker, Cybersecurity, 2. Aufl. 2022 (erscheint voraussichtlich im 1. Halbjahr 2022), Kap. 16, Rdnr. 262; vgl. auch Grzesiek, GuP 2021, 171, $175 \mathrm{ff}$. mit Hinweisen auf ergänzende Maßnahmen. Auch die bisher gültige „Technische Anlage“ zu den Hinweisen und Empfehlungen zur ärztlichen Schweigepflicht, Datenschutz und Datenverarbeitung in der Arztpraxis von BÄK und KBV hatte diese Aspekte zum Teil noch abgebildet und daher einen breiteren Blick auf die Cybersicherheit.

98) Grzesiek, GuP 2021, 171, $175 \mathrm{ff}$.

99) 22 Abs. 2 S. 2 Nr. 7 BDSG.

100) S.o. IV., 2., a).

101) S. o. bei III.

102) S. o. bei IV., 1., a).

103) Vgl. \$1 Abs. 2 S. 1 und 2 BDSG. Für die DSGVO gilt das wegen ihres Anwendungsvorrangs nur, soweit Öffnungsklauseln einen nationalen Rechtsgestaltungsraum gewähren.

104) Vgl. zu den noch nicht näher ausgeleuchteten Bereichen im Überblick Dochow/Herpers/Raptis, in: Kipker, Cybersecurity, 2. Aufl. 2022 (erscheint voraussichtlich im 1. Halbjahr 2022), Kap. 16, Rdnrn. $21 \mathrm{ff}$.

105) BSI, Die Lage der IT-Sicherheit in Deutschland 2021, S. 51.

106) S. o. bei IV., 1., a).

107) S. o. bei III.

108) Verordnung (EU) 2017/745 des Europäischen Parlaments und des Rates über Medizinprodukte v. 5.4.2017 (ABl. L 117 v. 5.5.2017, S. 1) - Medizinprodukteverordnung (MDR) und Medizinprodukterecht-Durchführungsgesetz (MPDG) mit ausführenden Rechtsverordnungen.

109) Zum Konformitätsbewertungsverfahren und zur CE-Kennzeichnung s. Art. 10 Abs. 5 MDR. 


\section{Digitale Gesundheitsanwendungen}

Digitale Gesundheitsanwendungen (DiGA) gem. IS 33 a 139e SGB V sind ,Medizinprodukte niedriger Risikoklasse, deren Hauptfunktion wesentlich auf digitalen Technologien beruht und die dazu bestimmt sind, bei den Versicherten oder in der Versorgung durch Leistungserbringer die Erkennung, Überwachung, Behandlung oder Linderung von Krankheiten oder die Erkennung, Behandlung, Linderung oder Kompensierung von Verletzungen oder Behinderungen zu unterstützen" ${ }^{110}$. Ein Anspruch des Versicherten besteht nur, soweit eine DiGA im Verzeichnis für digitale Gesundheitsanwendungen des Bundesinstituts für Arzneimittel und Medizinprodukte (BfArM) aufgenommen wurde ${ }^{111}$. Voraussetzung für eine Aufnahme in das DiGA-Verzeichnis sind u. a. Anforderungen an die Datensicherheit nach dem ,Stand der Technik“112. Die technische Richtlinie des BSI TR-03161 vom 15.4.2020 beschreibt zwar Sicherheitsanforderungen an DiGA. Diese soll aber nicht Voraussetzung für die Aufnahme ins DiGA-Verzeichnis $\operatorname{sein}^{113}$, was verwundert, weil sie den Stand der Technik abbildet. Damit ergeben sich nähere Vorgaben für die „Datensicherheit" "114 aus $₫ 4$ Abs. 1 und Abs. 6 i. V. mit Anlage 1 DiGAV ${ }^{115}$. Dazu gehören Selbsterklärungen des Herstellers zur Erfüllung der Anforderungen auf Basis des Fragebogens nach Anlage 1 DiGAV. Dies ist ein Katalog von Sicherheitsanforderungen im Rahmen des Zulassungsverfahrens. Der Fragebogen umfasst Basisanforderungen für alle DiGA und Zusatzanforderungen für DiGA mit sehr hohem Schutzbedarf. Seit 1.1.2022 kann das BfArM vom DiGAHersteller zum Nachweis der Erfüllung der Anforderungen an die Informationssicherheit auch die Vorlage eines Zertifikats einer von der Deutschen Akkreditierungsstelle $\mathrm{GmbH}$ (DAkkS) als Beleg verlangen ${ }^{116}$. Ab dem 1.1.2023 müssen DiGA die vom BSI festzulegenden Anforderungen erfüllen $^{117}$ und diese durch Vorlage eines Zertifikates nachweisen. Bis Nachweise in Form von Zertifikaten erbracht werden können, kann das BfArM auch die Vorlage von Prüfberichten zu Penetrationstests oder Sicherheitsgutachten unabhängiger Dritter verlangen, welche die Sicherheit der DiGA einschließlich aller Backend-Komponenten und Dienste umfassen. Selbsterklärungen sollen perspektivisch durch Zertifizierungsverfahren abgelöst werden ${ }^{118}$.

\section{Telemedizin}

Die im SGB V einschlägigen Rechtsgrundlagen für die Telemedizin, hier am Beispiel von Videosprechstunden, enthalten ebenfalls Anhaltspunkte für die Sicherstellung von Cybersicherheit. $₫ 365$ Abs. 1 S. 1 SGB V überträgt es den Selbstverwaltungspartnern , die Anforderungen an die technischen Verfahren zu Videosprechstunden, insbesondere Einzelheiten hinsichtlich der Qualität und der Sicherheit, und die Anforderungen an die technische Umsetzung" festzulegen. In den konkretisierenden untergesetzlichen Regelungen, namentlich der „Vereinbarung über die Anforderungen an die technischen Verfahren zur Videosprechstunde gem. $\ 365$ Abs. 1 SGB V“ (Anlage 31b BMV-Ä), finden sich daher auch „Bestimmungen zur Informationstechniksicherheit" $(\mathbb{} 2)^{119}$. Allgemein ist danach zu gewährleisten, ,,dass die erforderlichen technischen und organisatorischen Maßnahmen eingehalten werden“ ( $\$ 2$ Abs. 1). Eine Übertragung der Videosprechstunde darf grundsätzlich nur über eine Peer-to-Peer-Verbindung erfolgen. Bei einer Abweichung haben ,geeignete technische und organisatorische Maßnahmen ein angemessenes Schutzniveau zu gewährleisten“ ( $\$ 2$ Abs. 2). Die Inhalte der Videosprechstunde müssen ,während des gesamten Übertragungsprozesses nach dem Stand der Technik Endezu-Ende verschlüsselt" sein (\$2 Abs. 3) und außerdem ist ein Zugriffsschutz geregelt, nach dem durch den Videodienstanbieter keine Inhalte eingesehen werden dürfen $(\mathbb{} 2$ Abs. 4). Ferner darf der Videodienst keine schwerwiegen- den Sicherheitsrisiken (z. B. OWASP-TOP 10 Katalog) aufweisen ( $\$ 2$ Abs. 5). In $\$ 5$ Anlage 31b BMV-Ä sind zudem „Anforderungen an den Videodienstanbieter" mit weiteren Verpflichtungen sowie Hinweise auf Zertifikatsnachweise geregelt. Insbesondere bedarf der Videodienstanbieter gem. $\$ 5$ Abs. 2 einer Zertifizierung durch eine nach ISO/IEC 17065 akkreditierte Stelle.

Die Einhaltung der Anforderungen der Anlage 31b BMV-Ä ist Voraussetzung für die Abrechnung solcher Leistungen nach dem Einheitlichen Bewertungsmaßstab (EBM). Insoweit wird Cybersicherheit auch hier in den Zusammenhang mit finanziellen Aspekten gestellt ${ }^{120}$.

\section{Cybersicherheit durch die Telematikinfrastruktur}

\section{Telematikinfrastruktur als Kritische Infrastruktur}

Die zunehmende Digitalisierung und Vernetzung vieler Prozesse der Informationsverarbeitung im Gesundheitswesen wird maßgeblich mit der Telematikinfrastruktur (TI) ${ }^{121}$ und ihren Anwendungen assoziiert. „Die Telematikinfrastruktur ist die interoperable und kompatible Informations-, Kommunikations- und Sicherheitsinfrastruktur, die der Vernetzung von Leistungserbringern, Kostenträgern, Versicherten und weiteren Akteuren des Gesundheitswesens sowie der Rehabilitation und der Pflege dient" ( $\$ 306$ Abs. 1 S. 2 SGB V) ${ }^{122}$. Sie umfasst versorgungswichtige oder gar lebenswichtige Anwendungen wie das eRezept, das Notfalldatenmanagement, den Medikationsplan oder die elektronische Patientenakte ${ }^{123}$. Sie ist künftig von großer Bedeutung für das Funktionieren des Gemeinwesens, zumal sie eine Vielzahl von Akteuren im Gesundheitswesen betrifft, wie vor allem (Zahn-)Arztpraxen, Krankenhäuser und Apotheken, die verpflichtet sind, sich an die TI anzubinden.

In diesem Rahmen verwundert es nicht, dass Aspekte der Cybersicherheit von herausragender Bedeutung sind. Ein Ausfall oder eine Beeinträchtigung der TI würde erhebliche Risiken für die Sicherheit und Funktionsfähigkeit der (öffentlichen) Gesundheitsversorgung, einschließlich einer Gefährdung von Patienten, bedeuten. $\$ 306$ Abs. 3 SGB V betont mit Blick auf den Datenschutz zudem, dass wegen des „besonderen Schutzbedarfs" von Gesundheitsdaten gem. Art. 9 DSGVO ein „hohes Schutzniveau“ sicherzustellen ist.

Die Telematikinfrastruktur ist vor diesem Hintergrund als eine Kritische Infrastruktur i.S.v. \$2 Abs. 10 BSIG einzuordnen. Allerdings wird sie vom Anwendungsbe-

110) \$33a Abs. 1 S. 1 SGB V.

111) S. \$139e SGB V.

112) 1139 e Abs. 2 S. 2 Nr. 2 SGB V.

113) BT-Dr. 19/27652, S. 109.

114) Gemeint ist auch die „Informationssicherheit“. S. im Kontext der IT-Sicherheit Schrahe/Städter, DuD 2020, 713 f., $717 \mathrm{ff}$.

115) Verordnung über das Verfahren und die Anforderungen zur Prüfung der Erstattungsfähigkeit digitaler Gesundheitsanwendungen in der gesetzlichen Krankenversicherung (Digitale Gesundheitsanwendungen-Verordnung - DiGAV) v. 8.4.2020 (BGBl. I S. 768).

116) $\$ 7$ Abs. 3 DiGAV.

117) $\$ 4$ Abs. 7 DiGAV.

118) $\$ 4$ Abs. 8 DiGAV.

119) S. dazu Dochow/Herpers/Raptis, in: Kipker, Cybersecurity, 2. Aufl. 2022 (erscheint voraussichtlich im 1. Halbjahr 2022), Kap. 16, Rdnrn. 21, 243 f.; Raptis, Der Krankenhaus-JUSTITIAR, 4/2018, $105 \mathrm{ff}$.

120) Zu Förderungen im Krankenhausbereich s. o. IV., 1., b.

121) S. näher Dochow, Grundlagen und normativer Rahmen der Telematik im Gesundheitswesen, 2017.

122) Näher seit der Geltung des PDSG Dochow, MedR 2021, 979 ff. sowie Kircher, GuP 2021, $1 \mathrm{ff}$

123) $\$ 334$ Abs. 1 SGB V. 
reich des BSIG und der KritisV ausgenommen, weil für sie eine sektorspezifische Bereichsausnahme gem. $₫ 8 \mathrm{~d}$ Abs. 2 Nr. 3 , Abs. 3 Nr. 3 BSIG greift ${ }^{124}$. Die bereichsspezifischen Regelungen sind mit ,vergleichbaren Anforderungen“"125 (nunmehr) in $\$ \$ 306 \mathrm{ff}$. (insb. $\$ 329 \mathrm{ff} . \mathrm{SGB} V)^{126}$ enthalten. Wegen ihrer Kritikalität ist eine Anlehnung an $\$ 8$ a Abs. 1 u. 3 sowie $₫ 8 \mathrm{~b}$ BSIG gegeben ${ }^{127}$. Die umfassenden technischen und verfahrensmäßigen Vorgaben erfüllen zudem die Vorgaben von Art. 14 Abs. 1 bis 3, Art. 15 und Art. 21 NIS-RL ${ }^{128}$

\section{Regelungen zur Cybersicherheit in der Telematikinfrastruktur}

a) Sicherheitsleistungen

Als zentrale Sicherheitsinfrastruktur des Gesundheitswesens soll die TI insgesamt eine sichere Umgebung für die Digitalisierung und Vernetzung des gesamten Gesundheitswesens bieten ${ }^{129}$. Dabei bietet sie Basisdienste - einschließlich der Dienste zur Gewährleistung der IT-Sicherheit - an, die von den verschiedenen Anwendungen im Rahmen der Plattform genutzt werden. $\mathrm{Zu}$ den Sicherheitsleistungen gehören beispielsweise Smartcards (eGK, eHBA, SMC-B) ${ }^{130}$, Digitale Identitäten ${ }^{131}$, Konnektoren, ein sicherer Internetzugang durch einen VPN-Zugangsdienst, die Public-KeyInfrastruktur, Verschlüsselungen, elektronische Signaturen und ein komplexes Zugriffskonzept ${ }^{132}$.

\section{b) Betriebsverantwortung}

Daneben ist für die Cybersicherheit die Betriebsverantwortung von Bedeutung. Die TI wird durch die gematik $\mathrm{GmbH}$ aufgebaut und maßgeblich (mit-) verantwortet. Sie hat die Gesamtverantwortung für den Betrieb der TI, was sich aus den $\$ 311$ und $\$ \$ 323 \mathrm{ff}$. SGB V ergibt ${ }^{133}$. Dabei obliegt ihr insbesondere die Festlegung von Rahmenbedingungen mit der „Erstellung der funktionalen und technischen Vorgaben" "134. Die Betriebsverantwortung ist dabei keineswegs auf den „Schutz personenbezogener Daten“135 begrenzt ${ }^{136}$. Vielmehr werden der gematik für die Cybersicherheit und andere Aspekte bedeutsame Aufgaben ${ }^{137}$ übertragen $^{138}$. Ein wichtiger Maßstab ist dabei die Gewährleistung der Sicherheit oder der Aufrechterhaltung der Funktionsfähigkeit der Telematikinfrastruktur ${ }^{139}$. Die gematik veröffentlicht hierzu Spezifikationen und Konzepte, die auch „Vorgaben für den sicheren Betrieb“ und „Rahmenbedingungen“ für Zulassungen enthalten ${ }^{140}$. Ferner erstellt die gematik ein Sicherheitskonzept und trifft Festlegungen für die „Datensicherheit" ${ }^{* 141}$. Sie vergibt öffentliche Aufträge ${ }^{142}$ für den Betrieb der TI und erteilt Zulassungen für kommerziell angebotene Komponenten, Anwendungen, Dienste und Anbieter nebst Erteilung von Bestätigungen für weitere Anwendungen ${ }^{143}$.

c) Zulassungs- und Bestätigungsverfahren

Die Regelungen zur Cybersicherheit in der TI umfassen ein Zulassungs- und Bestätigungsverfahren. Das betrifft die Zulassung von Betriebsleistungen ( $\$ 324$ SGB V), z.B. des gesicherten Netzes, die Zulassung von Komponenten und Diensten ( $\$ 325$ SGB V) und die Bestätigung von weiteren Anwendungen ( $\$ 327$ SGB V) durch die gematik. Ein wesentliches Ziel von Zulassungen und Bestätigungen ist der Nachweis von Produktsicherheit und damit der Nachweis von Cybersicherheit.

Die Regelungen zur Zulassung von Komponenten und Diensten ( $\$ 325$ SGB V) sind in dem marktoffenen System eine wichtige Sicherheitsmaßnahme, weil hieraus bestimmte Vorgaben für Hersteller und Anbieter resultieren. Die gematik legt diese verbindlichen Rahmenbedingungen z.B. durch „Prüfkriterien“ und das „Nähere zum Zulassungsverfahren" fest ${ }^{144}$. Die Zulassung gem. \325 Abs. 1 erfolgt auf Antrag des Anbieters ${ }^{145}$. Dabei besteht ein Rechtsanspruch auf Zulassung, wenn Komponenten und Dienste gemäß der veröffentlichten Prüfkriterien funktionsfähig, interoperabel und sicher sind. Der Nachweis der Sicherheit erfolgt in der Regel durch eine Sicherheitszertifizierung nach den Vorgaben des BSI ${ }^{146}$. Die Zulassungsentscheidung ist ein Verwaltungsakt, der mit Nebenbestimmungen versehen werden kann ${ }^{147}$. Die Nutzung nicht zugelassener Komponenten und Dienste ist verboten ${ }^{148}$ und wird mit einem Bußgeld von bis zu dreihunderttausend Euro bedroht ${ }^{149}$.

Ferner ist ein Bestätigungsverfahren für ,weitere Anwendungen" vorgesehen ( $\$ 327$ SGB V). Diese Anwendungen nutzen die TI zwar (z.B. KV-eTerminservice), sind aber keine Komponenten der TI und werden deshalb nicht von der gematik zugelassen. Es handelt sich um Anwendungen des Gesundheitswesens, der Rehabilitation, der Pflege oder um eine Anwendung zum Zwecke der Gesundheits- und Pflegeforschung. Sie können über die TI verwendet werden und bei Bedarf Dienste der TI nutzen, wenn die Voraussetzungen von $\$ 327$ Abs. 1 SGB V vorliegen. Sie nutzen die TI mit ihren zentralen Diensten ${ }^{150}$ oder kryptographischen Identitäten letztlich als Plattform zur sicheren Vernetzung. Voraussetzungen für die Bestätigung gem. \327 SGB V ist v. a., dass die Sicherheit der TI nicht beeinträchtigt wird und die Datensicherheit in der Anwendung gewährleistet ist ${ }^{151}$. Das Nähere zu den erforderlichen Voraussetzungen legt die gematik fest und veröffentlicht diese Voraussetzungen auf

124) Köhler, GesR 2017, $145 \mathrm{f}$.

125) BT-Dr. 18/4096, S. 29; vgl. Köhler, GesR 2017, 145 f.; s.a. BReg., BT-Dr. 18/6990, S. 5: „dem BSI-Gesetz gleichwertigen Regelungen".

126) Die rechtliche Grundkonzeption wurde schon mit dem GMG im Jahr 2004 angelegt (s. \$219b Abs. 6, 7 und Abs. 8 und \$307 SGB V a.F.). Nach zwischenzeitlichen Anpassungen von $\$ 219 \mathrm{~b}$ SGB V a. F. an die NIS-R L erfolgte zuletzt mit dem PDSG eine systematische Neugestaltung im SGB V; s. dazu Dochow, MedR 2020, 979 f.

127) Köhler, GesR 2017, $145 \mathrm{f}$.

128) BT-Dr. 18/11242, S. 56, s. a. zum Entwurf der NIS-2-RL Dittrich/Dochow/Ippach, GesR 2021, 613, $621 \mathrm{ff}$.

129) Vgl. BT-Dr. 19/18793, S. 107.

130) S.z.B. $\$ 291$ Abs. 2 und $\$ 340$ SGB V.

131) S. $\$ 291$ Abs. 8 und $\$ 340$ Abs. $6-8$ SGB V.

132) Z.B. $\$ 352$ SGB V für die elektronische Patientenakte.

133) Grundsätzlich wird die Telematikinfrastruktur nicht von der gematik betrieben (BT-Dr. 18/5293, S. 49), sondern es werden dafür Dritte herangezogen ( $\$ 5323 \mathrm{ff}$. SGB V). Zur Ausnahme s. $\$ 323$ Abs. 2 S. 3 SGB V. Zu den Aufgaben der gematik s. ausf. Dochow/Herpers/Raptis, in: Kipker, Cybersecurity, 2. Aufl. 2022 (erscheint voraussichtlich im 1. Halbjahr 2022), Kap. 16 , Rdnrn. 35 ff.; s. a. Dochow, MedR 2020, 979, 985 f.

134) $\$ 311$ Abs. 1 Nr. 1 lit. a und Abs. 2 SGB V. Beschlüsse der gematik zu den Regelungen, dem Aufbau und dem Betrieb der TI sind u.a. für die Leistungserbringer und die Krankenkassen verbindlich ( $\$ 315$ Abs. 1 SGB V).

135) Dazu freilich $\$ 306$ Abs. 3 SGB V und $\$ 311$ Abs. 4 S. 1

136) A.A. Scholz, in: BeckOK Sozialrecht, $\$ 323$ SGB V, Rdnr. 2; dag. zutr. auf die missverständliche Lesart hinweisend Schifferdecker, in: KassKomm Sozialversicherungsrecht, 115. EL Juli 2021, $\$ 323$ SGB V, Rdnr. 3.

137) Z.B. die Gewährleistung von Diskriminierungsfreiheit und Barrierefreiheit, $\$ 311$ Abs. 1 Nr. 7, Abs. 4 S. 2 SGB V.

138) Vgl. etwa $₫ 311$ Abs. 4 S. 2 SGB V: ,zur Schaffung einer interoperablen, kompatiblen und sicheren Telematikinfrastruktur".

139) $₫ 323$ Abs. 2 S. 3 SGB V; vgl. auch $₫ 324$ Abs. 2 und $\$ 325$ Abs. 3 SGB V.

140) 3311 Abs. 1 Nr. 1 lit. c, Nr. 2, \$323 Abs. 1 SGB V.

141) $\$ 311$ Abs. 1 Nr. 1 lit. a, Abs. 2 SGB V.

142) $\$ \$ 307$ Abs. 3,323 Abs. 2 SGB V.

143) S. $\int \$ 323 \mathrm{ff}$. SGB V und die nachfolgenden Ausführungen.

144) $\$ 325$ Abs. 3 S. 1 u. 5 SGB V.

145) $\$ 325$ Abs. 1 S. 1 SGB V.

146) $\$ 325$ Abs. 3 S. 2 SGB $V$.

147) $\$ 325$ Abs. 2 S. 2 SGB V.

148) \$326 SGB V.

149) $\$ 397$ Abs. 2a Nr. 1 SGB V.

150) Z.B. der Verzeichnisdienst gem. $\$ 313$ SGB V.

151) $₫ 327$ Abs. 1 Nr. 2 SGB V. 
ihrer Internetseite ${ }^{152}$. Der Nachweis hat im Bestätigungsverfahren durch den Anbieter zu erfolgen ${ }^{153}$. Auf seinen Antrag hin ergeht ein Verwaltungsakt über die Bestätigung, der mit Nebenbestimmungen versehen werden kann ${ }^{154}$. Die Nutzung nicht bestätigter Anwendungen ist ebenfalls verboten ${ }^{155}$ und wird mit einem Bußgeld bedroht ${ }^{156}$.

\section{d) Sicherheitsverantwortung}

Die gematik hat neben der Betriebsverantwortung die Gesamtverantwortung für die Sicherheit des Betriebs der TI (SS $329 \mathrm{ff}$. SGB V) ${ }^{157}$. Dabei stehen ihr umfängliche Befugnisse zur Verfügung. Dazu zählen insbesondere Maßnahmen zur Gefahrenabwehr ( $\$ 329$ SGB V), Vorkehrungen zur Vermeidung von Störungen $\left(\mathbb{S} 330\right.$ SGB V) ${ }^{158}$ und Maßnahmen zur Betriebsüberwachung $(\$ 331 \text { SGB V })^{159}$. Zudem werden Anforderungen an die Dienstleister formuliert, welche die dezentralen Komponenten der Leistungserbringer warten $(\$ 332 \text { SGB V })^{160}$.

Die Abwehr von Gefahren für die Funktionsfähigkeit und Sicherheit der TI durch Komponenten und Dienste erfolgt in einem Gefüge aus Meldepflichten und Abwehrmaßnahmen. Anbieter haben es der gematik zu melden, soweit erhebliche Störungen der Verfügbarkeit, Integrität, Authentizität und Vertraulichkeit der Komponenten oder Dienste der TI auftreten ( $\$ 329$ Abs. 2 S. 1 SGB V). Erheblich sind gem. \329 Abs. 2 S. 2 SGB V Störungen, die zum Ausfall oder zur Beeinträchtigung der Sicherheit oder Funktionsfähigkeit der Komponenten oder Dienste oder zum Ausfall oder zur Beeinträchtigung der Sicherheit oder Funktionsfähigkeit der TI oder wesentlicher Teile führen können oder bereits geführt haben. In dem Meldesystem werden Sicherheitsvorfälle nicht direkt dem zuständigen BSI gemeldet, sondern im „Umweg“ über die gematik. Die gematik hat insoweit ihrerseits dem BSI eine von ihr als solche bewertete Gefahr für die „Funktionsfähigkeit oder Sicherheit der Telematikinfrastruktur“, die von den „Komponenten und Diensten“" ausgeht, zu melden ( $\$ 329$ Abs. 1 S. 2 SGB V). Außerdem hat sie dem BSI Störungen bezogen auf die Komponenten oder Dienste sowie weitere bedeutende Störungen mit ,,beträchtlichen Auswirkungen auf die Sicherheit oder Funktionsfähigkeit der Telematikinfrastruktur" (\$329 Abs. 4 SGB V) zu melden. Die Meldungen sind jeweils ,unverzüglich“ vorzunehmen und Unterlagen ,innerhalb von zwei Wochen“ vorzulegen ${ }^{161}$. Anbieter, die vorsätzlich oder fahrlässig eine Meldung gem. \329 Abs. 2 S. 1 SGB V nicht, nicht richtig, nicht vollständig oder nicht rechtzeitig vornehmen, begehen gem. $₫ 397$ Abs. 2a Nr. 2 SGB V eine Ordnungswidrigkeit, die mit einem Bußgeld von ,bis zu dreihunderttausend Euro" geahndet werden kann ${ }^{162}$.

Im Rahmen der Abwehrmaßnahmen ist die gematik verpflichtet und ermächtigt, unverzüglich die erforderlichen technischen und organisatorischen Maßnahmen zur Abwehr von Gefahren nach dem Stand der Technik zu ergreifen ( $\$ 329$ Abs. 1 S. 1 SGB V). Eine Gefahr ist dabei die eingetretene oder drohende erhebliche Beeinträchtigung der IT-Systeme ${ }^{163}$. Eine solche Gefahr kann von Diensten innerhalb und außerhalb der TI ausgehen ${ }^{164}$. Mögliche Maßnahmen sind in $\$ 329$ Abs. 3 SGB V aufgeführt. Mittels hoheitlicher Anordnungen von Maßnahmen kann die gematik z.B. Komponenten und Dienste für den Zugang zur TI sperren oder den weiteren Zugang von Bedingungen abhängig machen ( $\$ 329$ Abs. 3 S. 1 SGB V) oder sie kann verbindliche Anweisungen an Anbieter von Diensten und Anwendungen zur Beseitigung oder Vermeidung von Störungen bzw. zur Beseitigung von Sicherheitsmängeln erteilen (\$329 Abs. 3 S. 2 bzw. $\ 333$ Abs. 3 SGB V) $)^{165}$. Vorsätzliche oder fahrlässige Zuwiderhandlungen gegen eine solche vollziehbare Anordnung stellen gem. \397 Abs. 2a Nr. 3 SGB V eine Ordnungswidrigkeit dar, die mit einem Bußgeld von ,bis zu dreihunderttausend Euro“ geahndet werden kann ${ }^{166}$. e) Zwischenfazit und Ausblick auf die geplante TI 2.0

Cybersicherheit in der TI erfolgt durch den Nachweis von Produktsicherheit (v. a. durch Zulassungen und Bestätigungen), Sicherheitsleistungen (z.B. durch Verschlüsselungen, PKI, VPN), eine Gewährleistung von Betriebssicherheit (v. a. durch Überwachung der Funktionsfähigkeit und Sicherheit; Gefahrenabwehrmaßnahmen), ein komplexes Zugriffskonzept und Sanktionen.

Auch praktisch bescheinigen Experten der - noch nicht vollends ins Leben gehobenen - TI nach einer Sicherheitsanalyse im Bereich Cyber- und Applikationssicherheit, u.a. einer Sicherheitsbewertung der Public-Key-Infrastruktur (PKI) sowie ein Penetrationstest des Verzeichnisdienstes und der VPN-Zugangsdienste, ein „,hohes Sicherheitsniveau“"167. $\mathrm{Zu}$ den geplanten Veränderungen der TI in Richtung einer „Telematikinfrastruktur 2.0“168 äußerte sich zuletzt auch das BSI. Es stellte fest, dass dies mit „erheblichen Eingriffen in die Sicherheitsarchitektur" verbunden sei und betont, dass die ,Säulen der IT-Sicherheit“ bei einer TI 2.0 im Interesse des bisherigen Sicherheitsniveaus erhalten bleiben sollten ${ }^{169}$.

\section{Fazit und Thesen}

Cybersicherheit - ebenso wie Cyber-Resilienz ${ }^{170}$ - wird im Gesundheitswesen künftig von außerordentlicher Be-

152) $₫ 327$ Abs. 4 SGB V.

153) $\$ 327$ Abs. 3 S. 1 SGB V.

154) $\$ 327$ Abs. 3 S. 2 u. 3 SGB V.

155) $\$ 326 \mathrm{SGB} \mathrm{V}$.

156) $\$ 397$ Abs. 2a Nr. 1 SGB V.

157) BT-Dr. 19/20708, S. 171

158) Z.B. Forderung von Nachweisen (z. B. Durchführung von Prüfungen und Audits).

159) Z.B. der Einsatz von geeigneten Systemen zur Erkennung von Störungen und Angriffen ( $\$ 331$ Abs. 3 SGB V); näher z.B. Schifferdecker, in: KassKomm Sozialversicherungsrecht, 115. EL Juli 2021, \$331 SGB V, Rdnrn. 3 ff.

160) Zum Nachfolgenden näher Dochow/Herpers/Raptis, in: Kipker, Cybersecurity, 2. Aufl. 2022 (erscheint voraussichtlich im 1. Halbjahr 2022), Kap. 16, Rdnrn. $111 \mathrm{ff}$.

161) 329 Abs. 1 S. 2 , Abs. 2, Abs. 4 und $₫ 333$ Abs. 1 Nr. 2 SGB V.

162) $\$ 397$ SGB Abs. 3 SGB V. Zuständige Verwaltungsbehörde ist dabei das BSI (Abs. 4).

163) BT-Dr. 19/18793, S. 107

164) Vgl. BT-Dr. 18/5293, S. 51.

165) Ferner bestehen Kompetenzen des BSI zur Überprüfung mit u.a. der Möglichkeit, der gematik verbindliche Anweisungen zu geben ( $\$ 333$ Abs. 2 SGB V).

166) $\$ 397$ SGB Abs. 3 SGB V.

167) Ärztezeitung, 19.1.2021, abrufbar unter: https://www.aerztezeitung.de/Wirtschaft/gematik-sieht-TI-auf-der-sicheren-Seite-416364.html?utm_term $=2021-01-20 \& u t m \_s o u r c e=2021$ 01-20-AEZ_NL_NEWSLETTER\&utm_medium=email\& tid=TIDP744638X7A92D89272ED4C698AC02C399CE8AF7FYI4\&utm_campaign=AEZ_NL_NEWSLETTER [Zugriff am 14.1.2022].

168) gematik, Arena für digitale Medizin, Whitepaper Telematikinfrastruktur 2.0 für ein föderalistisch vernetztes Gesundheitssystem, Stand Dezember 2020, abrufbar unter: https://www. gematik.de/fileadmin/user_upload/gematik/files/Presseinformationen/gematik_Whitepaper_Arena_digitale_Medizin_ TI_2.0_Web.pdf [Zugriff am 14.1.2022].

169) Näher BSI, Die Lage der IT-Sicherheit in Deutschland 2021, S. 53; zusf. auch Grätzel von Grätz, EHealth.COM v. 27.10.2021, abrufbar unter: https://e-health-com.de/details-news/neuerbsi-bericht-it-sicherheit-angespannt-bis-kritisch/.

170) Dies bedeutet, dass zentrale Prozesse in Gesundheitseinrichtungen auch unter außergewöhnlichen Umständen, wie Sicherheitsvorfällen, funktionsfähig bzw. im Ernstfall schnell wiederherstellbar sein müssen (vgl. Kammerloher, DuD 2021, 649, 650 f.; s.a. Dittrich/Dochow/Ippach, GesR 2021, 613, 624 zum Entwurf einer Resilienz-Richtlinie der EU). Cyber-Resilienz steht damit in engem Zusammenhang mit Cybersicherheit. 
deutung sein, denn die forcierte Digitalisierung und Vernetzung bringt neuartige Risiken mit sich. Cybersicherheit darf daher nicht länger als lästiger Kostenfaktor verstanden werden, sondern ist Investition in eine sichere und stabil funktionierende Arbeitsumgebung in den Gesundheitseinrichtungen und damit zugleich eine solide Basis für eine erfolgreiche Digitalisierung im Gesundheitswesen.

Das Cybersicherheitsrecht im Gesundheitswesen ist zum Teil ein Resultat der jüngeren Rechtsentwicklung, auch wenn seine Verankerung im Datenschutzrecht historisch weiter zurückreicht. Wie im Datenschutzrecht besteht eine starke Fragmentierung der rechtlichen Grundlagen aufgrund der vielen rechtlichen Ebenen und bereichsspezifischen, z.T. nur untergesetzlichen Regelungen. Mit den einschlägigen Regelungen zur Telematikinfrastruktur werden hohe Standards gesetzt. Neben dem Datenschutz ist die IT- und Inforationssicherheit ein wesentlicher Eckpfeiler für die TI. Das ergibt sich aus dem bereichsspezifisch ausdifferenzierten Regelungssystem aus Zulassung, Betriebs- und Sicherheitsverantwortung. In den Sektoren des Gesundheitswesens fehlt zum Teil noch ein vergleichbarer ganzheitlicher Blick auf die Cybersicherheit, soweit etwa für Arztpraxen in den einschlägigen Regelwerken nur die IT-Sicherheit adressiert wird.
Cybersicherheitsrecht ist ungeachtet dessen kein nationales Phänomen. Längst weitet sich der Blick mindestens in Richtung Europäischer Union. Die EU-Kommission hat bereits im Dezember 2020 eine Cybersicherheitsstrategie „The EU's Cybersecurity Strategy for the Digital Decade" mit einem Maßnahmenpaket zur Stärkung des Schutzes kritischer Infrastrukturen und Stärkung der Widerstandsfähigkeit kritischer Infrastrukturen vorgestellt ${ }^{171}$. Das Paket enthält einen Vorschlag für eine weiterentwickelte Richtlinie über Maßnahmen für ein hohes gemeinsames Cybersicherheitsniveau in der Union (,NIS2-Richtlinie“" $)^{172}$ und eine neue Resilienz-Richtlinie ${ }^{173}$. Mit Abschluss der europäischen Rechtssetzung werden im nationalen Recht weitere Anpassungen erfolgen, die auch Bedeutung für das Gesundheitswesen haben werden ${ }^{174}$ und die Dynamik der Entwicklungen im Cybersicherheitsrecht verdeutlichen.

171) Europäische Kommission v. 16.12.2020, JOIN(2020) 18 final, abrufbar unter: https://ec.europa.eu/newsroom/dae/document. cfm?doc_id=72164 [Zugriff am 14.1.2022].

172) $\operatorname{COM}(2020) 823$ final.

173) $\operatorname{COM}(2020) 829$ final.

174) Näher Dittrich/Dochow/Ippach, GesR 2021, 613 ff.

\section{Die Überkreuzlebendspende (Cross over)}

\section{Bernd-Rüdiger Kern*}

Über die Zulässigkeit der Überkreuzspende nach dem geltenden Recht wird gestritten. Drei Meinungen lassen sich ausmachen. Die Überkreuzspende wird einerseits für unzulässig gehalten und andererseits nur dann für zulässig gehalten, wenn ein Näheverhältnis besteht oder aufgebaut wird. Diese beiden Ansichten dominieren die Diskussion. Als drittes kommt die direkte Anwendung von $\$ 8$ TPG ins Gespräch. Das Bundesministerium für Gesundheit hat am 29.6.2021 ein Symposion unter dem Titel: „Erweiterung des Spenderkreises bei der Lebendorganspende - eine Perspektive für Deutschland? Chancen und Risiken von cross-over-Lebendspenden, Pool-Spenden oder nicht gerichteten Lebensspenden in Deutschland“ veranstaltet. Bezüglich der Überkreuzspende war das Ergebnis in hohem Maße unbefriedigend. Das gibt Anlass, sich erneut mit dem Gesetzestext auseinanderzusetzen. Das unterblieb - bei den Vertretern der Mehrheitsmeinungen - merkwürdigerweise bisher weithin.

\section{Einführung}

Die Lebendspende ist als Möglichkeit in $\$ 8$ TPG geregelt, wurde aber auch schon vor 1997 in Deutschland durchgeführt ${ }^{1}$, obwohl es sich für den Spender nicht um einen Heileingriff handelt ${ }^{2}$. In einzelnen Fällen kann sich eine Lebendspende aus medizinischen Gründen aber als unmöglich oder jedenfalls nachteilig erweisen. Bei HLAAntigen- oder Blutgruppen-Inkompatibilität kann es zu starken Abstoßungsreaktionen beim Empfänger kommen.

Prof. Dr. iur. Bernd-Rüdiger Kern,

Institut für Recht und Ethik in der Medizin,

Lange Reihe 33, 04299 Leipzig, Deutschland
Diese können zwar durch entsprechende Vorbehandlungen vermieden oder überwunden werden; das lässt aber teilweise weniger gute Ergebnisse und eine erhöhte Rate an Komplikationen/Nebenwirkungen für den Empfänger erwarten $^{3}$. Zudem belastet eine solche Behandlung die Versichertengemeinschaft weitaus stärker.

Dieses Problem kann medizinisch durch eine Überkreuzspende gelindert oder ganz gelöst werden. Überkreuzspende bedeutet, dass zwei Paare nicht dem jeweiligen Partner spenden, sondern dem Partner des anderen Paares. Der spendebereite Partner des Paares A spendet an den Organpatienten des Paares B, und der spendebereite Partner des Paares B spendet an den potentiellen Organempfänger des Paares $\mathrm{A}^{4}$. Grundsätzlich ist auch der Austausch zwischen drei und mehr Paaren denkbar und medizinisch sinnvoll (Dominospende). Die Überkreuzspende verfolgt das Ziel, die Paare so auszusuchen, dass es zu möglichst wenigen Abstoßungsreaktionen beziehungsweise zum bestmögli-

^) Prof. Dr. iur. Bernd-Rüdiger Kern hatte bis zu seiner Emeritierung (2014) an der Universität Leipzig den Lehrstuhl für Bürgerliches Recht, Rechtsgeschichte und Medizinrecht inne.

1) Im Jahre 1994 stammten $4 \%$ der transplantierten Nieren von Lebendspendern; vgl. dazu Kühn, MedR 1998, S. 455, 458. Zur älteren Rechtslage vgl. Kern, Zivilrechtliche Gesichtspunkte der Transplantation, in: Gramberg-Danielsen, Rechtliche Grundlagen der augenärztlichen Tätigkeit, 1988, S. 2/800; und Uhlenbruck, Die zivilrechtliche Problematik der Organtransplantation, in: Laufs/ Uhlenbruck, Handbuch des Arztrechts, 1. Aufl. 1992, S. $768 \mathrm{ff}$

2) Schreiber/Wolfslast, MedR 1992, 189, 193.

3) Vgl. dazu Donauer, Hessisches Ärzteblatt 2006, 715 f. Der Verfasser dankt Prof. Dr. med. Christian Hugo (Universität Klinik Dresden) für weitere Hinweise.

4) Plastisch beschrieben bei Ulsenheimer, in: Laufs/Kern/Rehborn, Handbuch des Arztrechts, 5. Aufl. 2019, S. 1712. 